Preprint typeset in JHEP style - HYPER VERSION

\title{
Non-commutative Weitzenböck geometry, gerbe modules, and WZW branes
}

\author{
Andreas Recknagel ${ }^{a}$ and Rafat R. Suszek ${ }^{b}$ \\ ${ }^{a}$ King's College London, Department of Mathematics, Strand, London WC2R 2LS, UK \\ ${ }^{b}$ Laboratoire de Physique, École Normale Supérieure de Lyon, 46 Allée d'Italie, \\ F-69364 Lyon, France \\ andreas.recknagel@kcl.ac.uk, rafal.suszek@ens-lyon.fr
}

ABSTRACT: We study the non-commutative matrix model which arises as the low-energy effective action of open strings in WZW models. We re-derive this fuzzy effective gauge dynamics in two different ways, without recourse to conformal field theory: The first method starts from a linearised version of the WZW $\sigma$-model, which is classically equivalent to an action of the Schild type, which in turn can be quantised in a natural way to yield the matrix model. The second method relies on purely geometric symmetry principles - albeit within the non-commutative spectral geometry that is provided by the boundary CFT data: we show that imposing invariance under extended gauge transformations singles out the string-theoretic action up to the relevant order in the gauge field. The extension of ordinary gauge transformations by tangential shifts is motivated by the gerbe structure underlying the classical WZW model and standard within Weitzenböck geometry - which is a natural reformulation of geometry to use when describing strings in targets with torsion.

KeYwords: Conformal field theory, WZW models, D-branes on group manifolds; matrix models; Schild action; curved non-commutative geometry, spectral triples; bundle gerbes, gerbe modules; torsion, teleparallelism. 


\section{Contents}

1. Introduction 1

2. Fuzzy matrix dynamics directly from the WZW $\boldsymbol{\sigma}$-model 5

2.1 From the linearised WZW model to the Schild action 5

2.2 A matrix model for D0-branes 8

2.3 The torsion geometry of the linearised $\boldsymbol{\sigma}$-model 9

3. WZW models and bundle gerbes 10

3.1 A lightning course on bundle gerbes 11

$\begin{array}{lll}3.2 & \text { Twisted gauge fields on } \mathcal{G}_{\boldsymbol{H}} \text {-branes } & 12\end{array}$

4. The spectral geometry of the BCFT $\quad 15$

4.1 The spectral data 15

4.2 Non-commutative gauge symmetries: ordinary and extended 18

5. Conclusions and outlook 21

A. Appendix 23

\section{Introduction}

Branes have become a central object in string theory, and one nice feature is that they can be studied from various points of view, including target space geometry as well as the worldsheet CFT. String backgrounds where both descriptions are available, among them group targets and, to some extent, Calabi-Yau manifolds, offer particularly interesting insights. Here we will concentrate on WZW models, which are non-trivial in that their targets are curved and carry torsion, but which are still highly symmetric and hence tractable. The study of open-string dynamics has led to a more prominent rôle of non-commutative geometry, at a more practical level through the analysis of effective actions, and at a more fundamental level when discussing the true nature of geometry in string theory. Again, WZW models offer an ideal playground to test ideas - as we will try here by emphasising the rôle of torsion within the NCG picture.

The object we will be concerned with in this article is the low-energy effective action of open strings in WZW models with compact simple and simply connected target Lie group $G$, as derived in [1, 2]; this can be written in a concise form as a matrix model for $X \in \operatorname{Mat}(N ; \mathbb{C})$,

$$
\mathcal{S}_{\mathrm{ARS}}[X]=\operatorname{tr}\left(-\frac{1}{4}\left[X_{a}, X_{b}\right]\left[X_{a}, X_{b}\right]+\frac{\mathrm{i}}{3} f_{a b c} X_{a}\left[X_{b}, X_{c}\right]+\mu \cdot \mathbf{1}_{N}\right),
$$


where $\mu$ is a constant and $a=1,2, \ldots, d \equiv \operatorname{dim} G$. A less compact, but ultimately more geometric and physical reformulation in terms of dynamic variables $A_{a} \in \operatorname{Mat}(N ; \mathbb{C})$ is obtained by introducing the objects

$$
X_{a}=: Y_{a}+A_{a}
$$

where the $N \times N$ matrices $Y_{a}$ furnish a representation of the Lie algebra $\mathfrak{g}$ of $G$,

$$
\left[Y_{a}, Y_{b}\right]=\mathrm{i} f_{a b c} Y_{c} .
$$

From the CFT point of view, the $Y_{a}$ are induced by the action of the horizontal Lie algebra $\mathfrak{g}$ of the current symmetry algebra $\widehat{\mathfrak{g}}_{\mathrm{k}}$ of the boundary theory on CFT primaries (see the Appendix). From the point of view of non-commutative geometry, the redefinition (1.2) marks $X_{a}$ as a so-called covariant coordinate, an object composed of an inner derivation $Y_{a}$ (the rigid NCG background) and a gauge field $A_{a}$ (a fluctuation of the background), which on the whole is covariant under the action of the unitary gauge group.

In terms of the gauge field $A_{a}$ on the brane, the action (1.1) indeed takes on a familiar form: It can be written as the sum

$$
\mathcal{S}_{\mathrm{ARS}}[A]=\mathcal{S}_{\mathrm{YM}}[A]+\mathcal{S}_{\mathrm{CS}}[A]
$$

of a Yang-Mills and a Chern-Simons term

$$
\mathcal{S}_{\mathrm{YM}}[A]=\frac{1}{4} \operatorname{tr}\left[F_{a b}(A) F_{a b}(A)\right], \quad \mathcal{S}_{\mathrm{CS}}[A]=-\frac{\mathrm{i}}{2} \operatorname{tr}\left[f_{a b c} C S_{a b c}(A)\right],
$$

with

$$
\begin{array}{r}
F_{a b}(A)=\mathrm{i}\left[X_{a}, X_{b}\right]+f_{a b c} X_{c}=\mathrm{i}\left[Y_{a}, A_{b}\right]-\mathrm{i}\left[Y_{b}, A_{a}\right]+\mathrm{i}\left[A_{a}, A_{b}\right]+f_{a b c} A_{c}, \\
C S_{a b c}(A)=A_{a}\left[Y_{b}, A_{c}\right]+\frac{2}{3} A_{a} A_{b} A_{c}-\frac{\mathrm{i}}{2} f_{a b d} A_{d} A_{c} .
\end{array}
$$

One recognises that the $Y_{a}$ take over the rôle of derivatives on flat D-brane world-volumes. In rewriting (1.1) in this form, the constant $\mu$ has been chosen proportional to the Casimir eigenvalue $Y_{a} Y_{a}$.

In [2], this action was derived starting from the algebraic boundary CFT description of untwisted maximally symmetric WZW branes, which are given by Cardy boundary states labelled by elements $\lambda \in P_{+}^{k}(\mathfrak{g})$ of the set of dominant integral affine weights of the KacMoody algebra $\widehat{\mathfrak{g}}_{\mathrm{k}}$ at level $\mathrm{k}$. The open-string states of interest are $A_{a} j_{-1}^{a} \psi_{l}(x)$, where $\psi_{l}$ is a boundary field from the $\mathfrak{g}$-multiplet associated with some $\widehat{\mathfrak{g}}_{\mathrm{k}}$-primary (so the $A_{a}$ are matrices of coefficients). As long as $0 \lesssim\|\lambda\| \ll k$, these open-string states become massless in the low-energy (or decoupling) limit

$$
\alpha^{\prime} \longrightarrow 0, \quad \mathrm{k} \longrightarrow \infty, \quad \sqrt{\alpha^{\prime} \mathrm{k}} \equiv \ell \longrightarrow \infty \text {. }
$$

To deduce the effective action for those strings, one needs to exploit detailed knowledge of WZW conformal field theories to compute all CFT $n$-point correlation functions of these physical open-string states (dressed by ghosts as usual) up to the relevant order $n$ 
(determined by the desired order in $\alpha^{\prime}$ ) in the "spacetime fields" $A_{a}$, and then has to integrate out the world-sheet moduli. This process is medium involved at the technical level, but the most unsatisfactory aspect of the computation is the absence of any guiding principle which would allow to "guess" the outcome, or at least to recognise the resulting effective action as "reasonable".

The main aim of this paper is to identify such guiding principles. In Sect.2, we will see that the effective action (1.3) arises from linearising and quantising a Schild-type worldsheet action for the WZW model (without adding special boundary terms and without "detour" via CFT), much in the same way as one can obtain the IKKT matrix model [3] from the $\sigma$-model for flat strings $\llbracket$. This method is technically completely straightforward, but remains somewhat mysterious conceptually.

In the sections to follow, we therefore take a closer look at the non-commutativity of the effective action (1.3). In the classical $\sigma$-model picture, the symmetry-preserving boundary states mentioned above correspond to branes whose world-volume is a conjugacy class in the group target [5]. The effective action captures the dynamics of the effective openstring excitations, gauge bosons and transverse scalars taking values in the (co)tangent and (co)normal bundles ${ }^{1}$ of the conjugacy class, respectively. The CFT findings suggest that the open strings "see" quantised world-volumes in the form of "fuzzy" conjugacy classes [1] - for $G=S U(2)$, these are the well-known fuzzy spheres - and the action (1.3) indeed is a gauge theory on such a non-commutative space - a rather special gauge theory at that, as we are going to discuss presently.

We will carry out, in Sect. 1, a systematic reconstruction of the effective fuzzy geometry of untwisted maximally symmetric D-branes and the associated gauge dynamics, employing general tools of spectral non-commutative geometry (NCG). We will show that the effective action derived from CFT is uniquely determined, among all extensions of the usual YangMills term $\mathcal{S}_{\mathrm{YM}}[A]$ up to order four in the gauge field, by postulating invariance under an extended gauge symmetry, comprising usual unitary gauge transformations as well as tangential shifts - the gauge potential for the latter is nothing but the Kalb-Ramond field $B$. This in particular leads to the fine-tuning between the coefficients of the Yang-Mills term and the Chern-Simons term in (1.3): both are separately invariant under standard gauge transformations (as first remarked in [7] for the case of $G=S U(2)$ ) but have mass terms for $A$ which cancel out (and thus admit moduli for rigid shifts of branes) only in the particular combination which also enjoys the extended shift symmetry.

We will point towards the natural classical origin of this extended symmetry in Sect. 3, which briefly reviews the rôle of bundle gerbes and gerbe modules in WZW models.

A comment is in order concerning the Born-Infeld action, which captures a great deal of information on how branes, viewed as classical submanifolds of the target manifold, react to their surrounding target-space geometry. Applied to Lie groups for targets and conjugacy classes for world-volumes, the Born-Infeld action allows to understand how the $B$-field flux is capable of balancing the force of gravitational collapse, leading to the emergence of stable higher-dimensional D-branes on $G$ [8, 9, 10, 11]. One can even "derive" the effective action (1.3) from the Born-Infeld action by restricting to a stack of D0-branes placed in the

\footnotetext{
${ }^{1}$ Strictly speaking, we are working with the complexified group, $G{ }^{\mathbb{C}}$, compare the remarks in [6].
} 
background with non-vanishing Kalb-Ramond field ${ }^{2}$. We do not, however, regard this as a satisfactory explanation of the specific form of the low-energy effective action (1.3) because the argument relies on inserting D0-branes by hand, while for higher-dimensional WZW branes the Born-Infeld action gives an intrinsically continuous world-volume geometry instead of a matrix model as required: It seems that the Born-Infeld approach is simply "too classical" from the outset.

Before we turn to identifying "reasons" why (1.1) or (1.3) is in fact a very natural effective action, let us introduce some of its further characteristics. Its equations of motion can be written as

$$
\left[X_{a},\left[X_{a}, X_{b}\right]-\mathrm{i} f_{a b c} X_{c}\right]=0, \quad \text { or } \quad\left[Y_{a}+A_{a}, F_{a b}(A)\right]=0 ;
$$

the second form simply means that $F$ is covariantly constant. These equations were examined in great detail in [12] (but see also [13, 14, 15]), where it was in particular shown that solutions of the form

$$
F_{a b}(A)=\Phi_{a b} \cdot \mathbf{1}_{N}, \quad \Phi_{a b}=-\Phi_{b a} \in \mathbb{C}
$$

precisely correspond to maximally symmetric WZW branes, labelled by a positive weight $\lambda \in P_{+}(\mathfrak{g})$ and associated, à la Kirillov, to the conjugacy class $\mathcal{C}_{\lambda} \subset G$ wrapped by the (classical) D-brane. The correspondence to single conjugacy classes holds as long as $N=n \cdot \operatorname{dim} V_{\lambda}$ is a multiple of the dimension of an irreducible $\mathfrak{g}$-module, where $n$ is the number of Chan-Paton labels in a stack of D-branes wrapping the same conjugacy class $\mathcal{C}_{\lambda}$. Using techniques established for the boundary CFT description of the Kondo effect [16], it was argued in [2, 12, 13] that one can reach superpositions of stacks over different conjugacy classes upon brane condensations, i.e. renormalisation-group flows. These condensations can in particular change the dimension of the conjugacy class: higher-dimensional WZW branes can be viewed as bound states of D0-branes.

The relation between (1.7) and maximally symmetric branes was established in [2, 12] by comparing the value of the effective action at such a configuration to the known $g$ factors (or boundary entropies) for Cardy boundary states. Note that the equations of motion (1.6) allow for other solutions, but since the action (1.3) was derived in conformal perturbation theory around a maximally symmetric boundary condition, it will only see perturbative renormalisation-group fixed points.

Any gauge field configuration can be split into a traceless and a trace part,

$$
A_{a}=A_{a}^{0}+A_{a}^{T}, \quad \text { with } \quad A_{a}^{T}:=\frac{1}{N} \operatorname{tr} A_{a} \cdot \mathbf{1}_{N} .
$$

Using the Bianchi identity for $F_{a b}$, it can be shown [12] that whenever $A_{a}$ solves the equations of motion (1.6) then $F_{a b}\left(A^{0}\right)=0$. If $A_{a}$ moreover satisfies the maximal symmetry condition (1.7) then $\Phi_{a b} \equiv f_{a b c} A_{c}^{T}$.

\footnotetext{
${ }^{2}$ The result follows trivially from the observation that there are no objects charged under the KalbRamond potential $B$ in the situation considered and hence the term of the lowest (linear) order in this field is necessarily proportional to its exterior derivative, i.e. the field strength $H$. This term augments the standard (flat-case) Yang-Mills term and thereby reproduces $\mathcal{S}_{\text {ARs }}$.
} 
What is more relevant for our purposes is to note that the split (1.8) is preserved by the following extended gauge symmetry of $\mathcal{S}_{\mathrm{ARS}}$, parametrised by standard gauge transformations $U \in S U(N)$ and scalar shifts given by $\Pi_{a} \in \mathbb{C}$ :

$$
A_{a} \longmapsto A_{a}^{U, \Pi}:=U^{\dagger} A_{a} U+\mathrm{i} U^{\dagger}\left[Y_{a}, U\right]+\Pi_{a} \cdot \mathbf{1}_{N} .
$$

Indeed, separating $A_{a}^{U, \Pi}$ into its traceless and trace parts, we obtain

$$
\begin{array}{r}
A_{a}^{0} \longmapsto U^{\dagger} A_{a}^{0} U+\mathrm{i} U^{\dagger}\left[Y_{a}, U\right], \\
A_{a}^{T} \longmapsto A_{a}^{T}+\Pi_{a} \cdot \mathbf{1}_{N},
\end{array}
$$

which identifies $A^{0}$ as an $S U(N)$ gauge field. The fields $A_{a}^{T}$, on the other hand, are scalars under the gauge symmetry $S U(N)$ and can be viewed, physically, as the translational moduli associated with rigid one-sided $G$-translations in the target. By selecting some maximally symmetric D-brane (i.e. some conjugacy class), this global symmetry is broken so that $A^{T}$ becomes a Goldstone vector boson.

\section{Fuzzy matrix dynamics directly from the WZW $\sigma$-model}

In this section, we establish a technically straightforward but nevertheless conceptually surprising relation between the microscopic description of closed-string propagation in the Lie-group target and the fuzzy matrix model (1.3). We start from the WZW world-sheet action for closed strings, linearise around constant maps, then pass to a classically equivalent action of the Schild type. Next, one can subject the dynamical variables of the latter to a natural quantisation procedure, involving a truncation to finitely many degrees of freedom on the way. In this manner, one obtains the effective action (1.3) for open strings attached to maximally symmetric branes in the WZW target. Boundary conditions (which were originally left unspecified) arise naturally as a consistency condition in the process of truncation quantisation.

This procedure is a generalisation of Yoneya's derivation of the IKKT matrix model action (the D-instanton analogue of the BFSS matrix model) from the classical worldsheet action [⿶. Our derivation in particular allows to see the connections between the symmetries of the WZW model and torsion geometry very clearly, as will be described in Sect.2.3.

\subsection{From the linearised WZW model to the Schild action}

We start on a closed Euclidean world-sheet $\Sigma$ with coordinates $\sigma=\left(\sigma_{1}, \sigma_{2}\right)$ and metric $\gamma=\gamma_{A B} \mathrm{~d} \sigma^{A} \mathrm{~d} \sigma^{B}$. The action for the level-k WZW model is

$\mathcal{S}_{\mathrm{WZW}}[g, \gamma]=-\frac{\mathrm{k}}{4 \pi \alpha^{\prime}} \int_{\Sigma} \mathrm{d}^{2} \sigma \sqrt{\operatorname{det} \gamma}\left(\gamma^{-1}\right)^{A B} \operatorname{tr}\left[\left(g^{-1} \partial_{A} g\right)\left(g^{-1} \partial_{B} g\right)\right]+\frac{\mathrm{ik}}{4 \pi \alpha^{\prime}} \int_{\Sigma} g^{*} \mathrm{~d}^{-1} \chi(g)$,

with

$$
\chi(g)=\frac{1}{3} \operatorname{tr}\left[\left(g^{-1} \mathrm{~d} g\right) \wedge\left(g^{-1} \mathrm{~d} g\right) \wedge\left(g^{-1} \mathrm{~d} g\right)\right], \quad g \in G .
$$


We expand the maps $g(\sigma)$ around some fixed group element $g_{0} \in G$, i.e. consider

$$
g(\sigma)=: g_{0} \cdot \mathrm{e}^{\mathrm{i} \theta^{a} \widetilde{X}_{a}(\sigma)}=g_{0} \cdot\left[e+\mathrm{i} \theta^{a} \widetilde{X}_{a}(\sigma)+\mathcal{O}\left(\ell^{-2}\right)\right],
$$

where $e \in G$ is the group unit, $\theta^{a}$ form an orthonormal basis of the tangent space of $G$ at $g_{0}$ (a one-sided translate of $T_{e} G \cong \mathfrak{g}$ ) and satisfy the standard structure relations

$$
\left[\theta^{a}, \theta^{b}\right]=\mathrm{i} f_{a b c} \theta^{c}
$$

the objects

$$
\widetilde{X}_{a} \equiv \frac{1}{\ell} \cdot X_{a},
$$

are dimension-less world-sheet embedding fields. The appearance of the stringy length scale $\ell=\sqrt{\alpha^{\prime} \mathrm{k}}$ makes it clear that the linearisation of the WZW action is in fact a long-distance or low-energy approximation.

Substituting the expansion (2.2) into (2.1) and keeping only terms up to the order $\ell^{-3}$, we obtain the linearised world-sheet model $^{3}$

$$
\mathcal{S}_{\operatorname{lin}}[X ; \gamma]=\mathcal{N} \int_{\Sigma} \mathrm{d}^{2} \sigma\left(\sqrt{\operatorname{det} \gamma}\left(\gamma^{-1}\right)^{A B} \delta_{a b} \partial_{A} X_{a} \partial_{B} X_{b}+\frac{\mathrm{i}}{3 \ell} \epsilon^{A B} f_{a b c} X_{a} \partial_{A} X_{b} \partial_{B} X_{c}\right)
$$

with a normalisation constant $\mathcal{N} \equiv \frac{1}{4 \pi \alpha^{\prime 2}}$.

In the next step, we apply arguments from [⿶凵⿴囗十 and establish classical equivalence between $\mathcal{S}_{\text {lin }}$ and the Schild-type action [17]

$$
\mathcal{S}_{\text {Schild }}[X ; e]=\int_{\Sigma} \mathrm{d}^{2} \sigma e\left(\frac{1}{4} \delta_{a c} \delta_{b d}\left\{X_{a}, X_{b}\right\}_{e}\left\{X_{c}, X_{d}\right\}_{e}+\frac{\mathrm{i}}{3 \ell} f_{a b c} X_{a}\left\{X_{b}, X_{c}\right\}_{e}+\mu\right),
$$

written in terms of an auxiliary field $e=e(\sigma)$ - which is a positive scalar density of weight $w(e)=-1$ on the world-sheet - , a positive constant $\mu>0$ and the so-called Nambu-Poisson bracket

$$
\{f, g\}_{e}:=\frac{1}{e} \epsilon^{A B} \partial_{A} f \partial_{B} g
$$

on the algebra of smooth functions on the world-sheet. The proof of the equivalence between $\mathcal{S}_{\text {lin }}$ and $\mathcal{S}_{\text {Schild }}$ proceeds by introducing a third, intermediate model

$$
\mathcal{S}_{\sim}[X ; e, t]=\int_{\Sigma} \mathrm{d}^{2} \sigma \frac{1}{e}\left(-\operatorname{det} t+\frac{t^{A B}}{\sqrt{2}} \delta_{a b} \partial_{A} X_{a} \partial_{B} X_{b}+\frac{\mathrm{i} e^{2}}{3 \ell} f_{a b c} X_{a}\left\{X_{b}, X_{c}\right\}_{e}+e^{2} \mu\right)
$$

with $e$ and $\mu$ as above and $t^{A B}=t^{B A}$ another auxiliary field (a tensor density of weight $w(t)=-2$ on the world-sheet). Passing to

$$
\widetilde{t}^{A B}:=t^{A B}-\frac{1}{\sqrt{2}} \epsilon^{A C} \epsilon^{B D} \delta_{a b} \partial_{C} X_{a} \partial_{D} X_{b},
$$

\footnotetext{
${ }^{3}$ The contribution of the kinetic term in $\mathcal{S}_{\mathrm{WZW}}$ trilinear in $X$ turns out to be traceless.
} 
we readily check the identity

$$
\mathcal{S}_{\sim}[X ; e, t] \equiv \mathcal{S}_{\text {Schild }}[X ; e]-\int_{\Sigma} \mathrm{d}^{2} \sigma \frac{\operatorname{det} \widetilde{t}}{e}
$$

and (classical) equivalence of these two models follows ${ }^{4}$ since the equation of motion for $\widetilde{t}^{A B}$ is simply $\widetilde{t}^{A B}=0$.

Next, we rescale the dynamical fields as $X \longmapsto \sqrt{\frac{\mu}{2}} \cdot X$ and perform a change of variables $(e, t) \longmapsto(\widetilde{e}, \gamma)$ in (2.6), with $\widetilde{e}$ a world-sheet scalar and $\gamma=\left(\gamma_{A B}\right)$ a metric tensor on $\Sigma$, such that

$$
e=e(\widetilde{e}, \gamma)=\widetilde{e} \sqrt{\operatorname{det} \gamma}, \quad t=t(\widetilde{e}, \gamma)=\left(\widetilde{e}^{2} \operatorname{det} \gamma\right) \cdot \gamma^{-1}
$$

In this way, we obtain

$$
\begin{gathered}
\mathcal{S}_{\sim}[X ; e, t]=\left(\frac{\mu}{2}\right)^{\frac{3}{2}} \int_{\Sigma} \mathrm{d}^{2} \sigma \sqrt{\operatorname{det} \gamma}\left(\frac{\widetilde{e}}{\sqrt{\mu}}\left(\gamma^{-1}\right)^{A B} \delta_{a b} \partial_{A} X_{a} \partial_{B} X_{b}+\frac{\mathrm{i}}{3 \ell} f_{a b c} X_{a}\left\{X_{b}, X_{c}\right\}_{\gamma}\right) \\
-\int_{\Sigma} \mathrm{d}^{2} \sigma \sqrt{\operatorname{det} \gamma} \widetilde{e}\left(\widetilde{e}^{2}-\mu\right)
\end{gathered}
$$

where now the Nambu-Poisson bracket is defined using $\gamma$,

$$
\{f, g\}_{\gamma}=\frac{1}{\sqrt{\operatorname{det} \gamma}} \epsilon^{A B} \partial_{A} f \partial_{B} g .
$$

Solving the equation of motion for the trace part of $\gamma$,

$$
\left(\gamma^{-1}\right)^{A B} \frac{\delta \mathcal{S}_{\sim}}{\delta\left(\gamma^{-1}\right)^{A B}} \stackrel{!}{=} 0
$$

we obtain

$$
\widetilde{e}=\sqrt{\mu}
$$

by virtue of the assumed positivity of $\widetilde{e}$. Plugging this result back into (2.7), we arrive at the classically equivalent model

$$
\overline{\mathcal{S}}_{\sim}[X ; \gamma]=\left(\frac{\mu}{2}\right)^{\frac{3}{2}} \int_{\Sigma} \mathrm{d}^{2} \sigma \sqrt{\operatorname{det} \gamma}\left(\left(\gamma^{-1}\right)^{A B} \delta_{a b} \partial_{A} X_{a} \partial_{B} X_{b}+\frac{\mathrm{i}}{3 \ell} f_{a b c} X_{a}\left\{X_{b}, X_{c}\right\}_{\gamma}\right)
$$

which reproduces - up to an irrelevant rescaling - the action (2.3),

$$
\overline{\mathcal{S}}_{\sim}[X ; \gamma] \equiv \frac{1}{\mathcal{N}}\left(\frac{\mu}{2}\right)^{\frac{3}{2}} \mathcal{S}_{\operatorname{lin}}[X ; \gamma]
$$

Thus, we have established the classical equivalence of the linearised WZW action and the Schild action,

$$
\mathcal{S}_{\text {Schild }}[X ; e] \sim \mathcal{S}_{\operatorname{lin}}[X ; \gamma]
$$

which leaves us the Schild model (2.4) to play with further.

\footnotetext{
${ }^{4}$ Strictly speaking, we have only proved the existence of a bijection between classical configurations of the two models. The latter compose the respective phase spaces which are endowed with additional structure - the (pre)symplectic structure, central to the quantisation of the models. The mapping discussed can actually be demonstrated to define a symplectomorphism between the two phase spaces, and it provides an example of a completely general symplectomorphic equivalence between the Polyakov and the Schild formulation valid for an arbitrary two-dimensional (dilaton-free) non-linear $\sigma$-model, discussed in 18 ].
} 


\subsection{A matrix model for D0-branes}

The crucial property of the Schild model is that it is formulated entirely in terms of the embedding field $X$ and its world-sheet Nambu-Poisson brackets (2.5). The latter admit a straightforward canonical quantisation

$$
\{\cdot, \cdot\}_{e} \longmapsto-\mathrm{i}[\cdot, \cdot]
$$

In addition, we make the replacement 19]

$$
\int_{\Sigma} \mathrm{d}^{2} \sigma e \longmapsto \operatorname{Tr}_{\mathcal{H}}
$$

of the world-sheet integral by a trace over the ensuing Hilbert space $\mathcal{H}$ - which is the standard replacement accompanying canonical quantisation: the integral with respect to the Liouville measure over the classical "phase space" to be quantised (here, it is simply the world-sheet $\Sigma$ equipped with the Nambu-Poisson structure) becomes the trace over the Hilbert space. So from the formal point of view, the transition (2.8) and (2.9) is tantamount to the canonical quantisation of the simple symplectic structure on $\Sigma$ associated with the Nambu-Poisson bracket (2.5) on the algebra $C^{\infty}(\Sigma)$ of smooth functions on the worldsheet.

We can now furthermore choose a "regularisation" by demanding that $\mathcal{H}$ is in fact finite-dimensional - which is a reasonable choice (and the typical outcome) for a compact phase space. This makes the $X$ into matrices and takes us from the original world-sheet model to a matrix model, namely

$$
\mathcal{S}_{M}[X]=\operatorname{Tr}_{\mathcal{H}}\left(-\frac{1}{4} \delta_{a c} \delta_{b d}\left[X_{a}, X_{b}\right]\left[X_{c}, X_{d}\right]+\frac{1}{3 \ell} f_{a b c} X_{a}\left[X_{b}, X_{c}\right]+\mu\right),
$$

which is, up to a rescaling $\left(X_{a}, \mu\right) \longmapsto\left(-\mathrm{i} \ell^{-1} X_{a}, \ell^{-4} \mu\right)$ and a trivial overall normalisation, nothing but the fuzzy matrix model (1.1),

$$
\mathcal{S}_{\mathrm{ARS}}[X] \equiv \ell^{4} \mathcal{S}_{M}[X]
$$

Let us now assume that the world-sheet $\Sigma$ has a boundary and define the original WZW action (2.1) there, without adding any boundary terms (which should, of course, be done to obtain a well-defined path integral formulation, see the brief review in Sect. 3 below). In this setting, the "regularisation" chosen above, which makes the $X$ into bounded operators, has some immediate consequences. The basic property of the trace

$$
\operatorname{Tr}_{\mathcal{H}}\left(\left[X_{a}, X_{b}\right]\right)=0
$$

translates back into an integral of the Poisson bracket, leading to the condition 20]

$$
\int_{\partial \Sigma} \mathrm{d} t X_{b} \partial_{t} X_{a}=0
$$

on the variables in the Schild action. Thus, one is led to Dirichlet conditions in all directions

$$
\left.\partial_{t} X_{a}\right|_{\partial \Sigma}=0
$$


as the natural (and probably the only admissible) choice of boundary conditions over the world-sheet boundary.

Note the difference between (2.10) and the boundary term of the variation in, say, a free boson theory on the upper half plane: the latter allows for both Dirichlet and Neumann boundary conditions. Note also that Dirichlet conditions in all directions are the ones that solve the boundary variation problem in any non-linear $\sigma$-model, while Neumann conditions are generically obstructed by the $B$-field.

Our little derivation leading from the world-sheet action of the WZW model to the open-string effective action in addition suggest that the latter is a model describing D0brane dynamics - completely in line with the CFT result that higher-dimensional branes can be obtained as bound states of D0-branes.

\subsection{The torsion geometry of the linearised $\sigma$-model}

So far, we have focused on the canonical and algebraic structure of the models involved, leaving aside the geometric interpretation of their building blocks - in particular of the metric and the $B$-field. While most of the following remarks are true in arbitrary conformally invariant $\sigma$-models, the linearised WZW action (2.3) offers a convenient starting point to discuss symmetries and to illuminate the geometric rôle of the Kalb-Ramond field $B$ from this perspective. The wider context of the ensuing interpretation is given by the "geometrostasis" ideas from [21]. For us, its main importance lies in the fact that it naturally leads to the notion of a torsion gerbe (present in all $\sigma$-models with a curved target, in particular WZW models), and lifting the differential-geometric structure of a bundle gerbe on the group manifold to the fuzzy régime will eventually shed new light on the effective open-string dynamics of WZW D-branes.

To get there, we first study symmetries of the model, as descended from those of its complete (group-integrated) version (2.1). It is easy to check that the symmetries which survive the linearisation are the tangent-space counterparts of the left-right shifts by group elements constant on the world-sheet. Thus, the left-right affine-algebra symmetry $\widehat{\mathfrak{g}}_{\mathrm{k}}^{L} \otimes \widehat{\mathfrak{g}}_{\mathrm{k}}^{R}$ of the WZW model reduces to its horizontal component, and its action splits into a vector (adjoint) part

$$
X_{a} t_{a} \equiv X \longmapsto X+\mathrm{i}[\Lambda, X]=\left(X_{a}+f_{a b c} X_{b} \Lambda_{c}\right) t_{a}, \quad \text { with } \quad \Lambda \equiv \Lambda_{a} t_{a} \in \mathfrak{g},
$$

and the one-sided $\mathfrak{g}$-shifts

$$
X \longmapsto X+\Pi, \quad \text { with } \quad \Pi \equiv \pi_{a} t_{a} \in \mathfrak{g}
$$

which come from one-sided group translations (neither $\Lambda$ nor $\Pi$ depend on the world-sheet variable $\sigma$ ). It is important to note that both symmetries are present in an unaltered form in the Schild action (2.4) as well.

For WZW models, the Cartan-Killing metric and the Kalb-Ramond field locally take the form [22, 23]

$$
\mathrm{g}_{a b}=\delta_{a b}+\mathcal{O}\left(\ell^{-2}\right), \quad B_{a b}=\frac{1}{3 \ell} f_{a b c} X_{c}+\mathcal{O}\left(\ell^{-3}\right)
$$


and to derive their transformation properties, we treat them as (tangent-space) tensors and so simply compute their Lie derivatives in the direction of the relevant variation vector fields,

$$
\delta_{\Lambda}^{\text {iso }} X_{a}=f_{a b c} X_{b} \Lambda_{c}, \quad \delta_{\Pi}^{\mathrm{axi}} X_{a}=\pi_{a} .
$$

(The notations "iso" and "axi" are borrowed from [21] and stand for the adjoint and shift symmetry, respectively.) In this way, we obtain

$$
\delta_{\Lambda}^{\text {iso }} \mathrm{g}_{a b}=0=\delta_{\Pi}^{\text {axi }} \mathrm{g}_{a b}, \quad \delta_{\Lambda}^{\text {iso }} B_{a b}=0,
$$

and

$$
\delta_{\Pi}^{\text {axi }} B_{a b}=-\partial_{[a} \widetilde{\pi}_{b]}, \quad \text { with } \quad \widetilde{\pi}_{a}=-\frac{1}{3 \ell} f_{a b c} X_{b} \pi_{c} .
$$

The lesson to draw from this simple calculation is that the torsion potential $B$ is a gauge field for tangential translations, under which it transforms simply as

$$
B \stackrel{\Pi}{\rightarrow} B-\mathrm{d} \widetilde{\Pi} .
$$

This is, indeed, the fundamental rôle of the torsion potential in gauge approaches to gravity, and - in particular - in the teleparallel gravity theory for so-called Weitzenböck geometries, which work with flat connections with torsion instead of (but equivalent to) the more familiar Levi-Civita connections. The latter fits perfectly with the effective equations of motion in string theory derived from the vanishing of the beta function (to first order in $\alpha^{\prime}$ ), which require (to have non-anomalous conformal symmetry) that the curvature is precisely cancelled by the torsion field $H$, for which the Kalb-Ramond field is a local potential; equivalently, one can augment the standard Levi-Civita connection of the metric $\mathrm{g}$ by the torsion field in such a way as to form a (Weitzenböck) connection with a vanishing Riemann tensor. It is this phenomenon of cancellation between the two contributions to the Riemann curvature, the (metric) Levi-Civita one and the (topological) torsion one, required by non-anomalous conformal symmetry of the underlying $\sigma$-model, which was termed "geometrostasis" in the original papers ${ }^{5}$ [21.

\section{WZW models and bundle gerbes}

The gauge freedom intrinsic to the definition of the torsion field above, and even the sheer presence of torsion, find their natural origin in the gerbe structure underlying the topological WZ term in the WZW action functional. Whenever one considers string targets with a non-trivial $B$-field, issues like gauge invariance require a more careful analysis, taking into account extra global structures in the form of a bundle gerbe [28, 29, 30]. In this section, we briefly review the necessity to consider gerbes in string theory, mainly following [28, 29, 30] and [31]. We also recall the defining properties of a bundle gerbe $\mathscr{G}_{H}$ : In the context of non-linear $\sigma$-models, bundle gerbes are the natural differentialgeometric structure induced in the target by the presence of a closed non-trivial torsion

\footnotetext{
${ }^{5}$ For further literature on teleparallel gravity, see [24, 25, 26]. Gauge theories of gravity were recently reviewed in [27].
} 
3-form $H$ (for which $B$ is a local potential). In direct analogy with line bundles, bundle gerbes comprise (Deligne) cohomology classes and the relevant cohomological equivalence (the so-called stable isomorphism) manifests itself in particular through the translational gauge symmetry discussed in the previous section. We will implant this bundle gerbe gauge symmetry, in Sect. 1 , into the non-commutative framework and arrive at a symmetry principle that singles out the low-energy effective action of open-string theory within NCG.

\subsection{A lightning course on bundle gerbes}

Let us start by recalling a few basic facts about bundle gerbes using their local description, as detailed, e.g., in [32]. Thus, a bundle gerbe $\mathscr{G}_{H}$ associated to the closed 3-form background $H$ (the globally defined curvature of $\mathscr{G}_{H}$ ) is - from the physical point of view - a construct $^{6}$ which enables us to give meaning to topological actions, of the kind of the WZ term in (2.1), in topologically nontrivial geometries $\mathcal{M}$. An example of such a geometry is a compact Lie group. Here, $\mathcal{M}$ is the target space of the $\sigma$-model, in which the world-sheet $\Sigma$ is embedded via

$$
\phi: \Sigma \longmapsto \mathcal{M}
$$

The obvious problem that we encounter when trying to give meaning to the WZ term is the lack of a global potential for $H$. The solution to this problem valid in all generality ${ }^{7}$ uses local data for the bundle gerbe, as captured by the Deligne cohomology group $H^{2}\left(\mathcal{M}, \mathcal{D}^{(2)}\right)$ based on the differential complex

$$
\mathcal{D}^{(2)}: \underline{U(1)} \stackrel{\frac{1}{\mathrm{i}} \mathrm{d} \log }{\longrightarrow} \underline{\Omega}^{1}(\mathcal{M}) \stackrel{\mathrm{d}}{\longrightarrow} \underline{\Omega}^{2}(\mathcal{M}) .
$$

Above, $U(1), \underline{\Omega}^{1}(\mathcal{M})$ and $\underline{\Omega}^{2}(\mathcal{M})$ are the sheaves of smooth $U(1)$-valued functions, smooth 1 -forms and smooth 2 -forms on $\mathcal{M}$, respectively. The local data are given by a family of triples $\left(B_{i}, \alpha_{i j}, g_{i j k}\right)_{i, j, k \in \mathcal{I}} \in \check{C}^{0}\left(\mathscr{U}, \underline{\Omega}^{2}\right) \oplus \check{C}^{1}\left(\mathscr{U}, \underline{\Omega}^{1}\right) \oplus \check{C}^{2}(\mathscr{U}, \underline{U(1)})$ of elements of the sets of $\check{C}$ ech $p$-cochains $\check{C}^{p}(\mathscr{U}, A)$ with values in the appropriate sheaves $A \in\left\{\underline{U(1)}, \underline{\Omega}^{1}, \underline{\Omega}^{2}\right\}$. The Cech cochains are defined in the standard way with reference to a good covering $\mathscr{U} \equiv\left\{\mathscr{U}_{i}\right\}_{i \in \mathcal{I}}$ of $\mathcal{M}$, that is an open covering such that all nonempty multiple intersections $\mathscr{U}_{i_{1} i_{2} \ldots i_{n}} \equiv \mathscr{U}_{i_{1}} \cap \mathscr{U}_{i_{2}} \cap \ldots \cap \mathscr{U}_{i_{n}} \neq \emptyset$ are contractible. A multi-indexed object $X_{i_{1} i_{2} \ldots i_{n}}$ is defined on the non-empty multiple overlap $\mathscr{U}_{i_{1} i_{2} \ldots i_{n}}$ and satisfies $X_{i_{\sigma(1)} i_{\sigma(2)} \ldots i_{\sigma(n)}}=\operatorname{sign}(\sigma) X_{i_{1} i_{2} \ldots i_{n}}$ for any permutation $\sigma \in \mathfrak{S}_{n}$ of the indices.

In terms of the local data, the gerbe can be viewed as a collection of so-called curvings $B_{i}$, connections $\alpha_{i j}$ and transition functions $g_{i j k}$, defined patch-wise and related by the

\footnotetext{
${ }^{6}$ Bundle gerbes were introduced by Giraud in [33] and later rephrased and developed by Brylinski in [32], where contact was made with the cohomological approach to WZW models due to Alvarez [31] and Gawȩdzki 28]. Strictly speaking, their application in the WZW setting requires a reformulation of the original concept in the more natural geometric language of Murray [34, 35], see also [36] for an introduction to the subject, but we shall not need the complete picture in what follows.

${ }^{7} \mathrm{~A}$ solution applicable in the case of a simply connected Lie-group target boils down to the introduction of a filling manifold for $\Sigma$, together with an appropriate extension of $\phi$, and was proposed already in the original paper [37. This prescription fails in the non-simply connected case.
} 
constraints

$$
\left\{\begin{array}{l}
\left.H\right|_{\mathscr{U}_{i}}=: \mathrm{d} B_{i} \\
\left.\left(B_{j}-B_{i}\right)\right|_{\mathscr{U}_{i j}}=: \mathrm{d} \alpha_{i j} \\
\left.\left(\alpha_{j k}-\alpha_{i k}+\alpha_{i j}\right)\right|_{\mathscr{U}_{i j k}}=: \mathrm{id} \log g_{i j k} \\
\left.\left(g_{j k l} g_{i k l}^{-1} g_{i j l} g_{i j k}^{-1}\right)\right|_{\mathscr{U}_{i j k l}} \stackrel{!}{=} 1
\end{array}\right.
$$

modulo the equivalences

$$
\left\{\begin{array}{l}
B_{i} \longrightarrow B_{i}-\mathrm{d} \widetilde{\Pi}_{i} \\
\left.\alpha_{i j} \longrightarrow\left(\alpha_{i j}-\widetilde{\Pi}_{j}+\widetilde{\Pi}_{i}+\mathrm{id} \log \widetilde{\chi}_{i j}\right)\right|_{\mathscr{U}_{i j}} \\
\left.g_{i j k} \longrightarrow\left(g_{i j k} \widetilde{\chi}_{j k} \tilde{\chi}_{i k}^{-1} \widetilde{\chi}_{i j}\right)\right|_{\mathscr{U}_{i j k}}
\end{array}\right.
$$

valid for any $\left(\widetilde{\Pi}_{i}, \widetilde{\chi}_{i j}\right)_{i, j \in \mathcal{I}} \in \check{C}^{0}\left(\mathscr{U}, \underline{\Omega}^{1}\right) \oplus \check{C}^{1}(\mathscr{U}, U(1))$. The main point of the local differential-geometric structure on $\mathcal{M}$ thus introduced is that it provides an unambiguous definition (i.e. it assigns a $c$-number value) to the topological WZ amplitude

$$
\mathrm{e}^{\mathrm{i} \int_{\Sigma} \phi^{*} \mathrm{~d}^{-1} H} \equiv \prod_{t \in \triangle_{\Sigma}} \mathrm{e}^{\mathrm{i} \int_{t} \phi_{t}^{*} B_{i_{t}}} \prod_{e \subset t} \mathrm{e}^{\mathrm{i} \int_{e} \phi_{e}^{*} \alpha_{i_{t} i_{e}}} \prod_{v \subset e \subset t} g_{i_{t} i_{e} i_{v}}^{\epsilon(v)}(\phi(v)) \equiv \operatorname{Hol}_{\mathcal{G}_{H}}(\phi),
$$

independent of all choices made as long as the world-sheet is closed, $\partial \Sigma=\emptyset$. Here, $\triangle_{\Sigma}=\{t, e, v\}$ is a triangulation of $\Sigma$ compatible with $\mathscr{U}$ in the sense that the plaquettes $t$, their edges $e$ and vertices $v$ are chosen such that for each of them, $f \in \triangle_{\Sigma}$, we have $\phi(f) \subset \mathscr{U}_{i_{f}}$ for some element of the covering. Whenever the orientation of $v$, as inherited from $t$ via $e$, is negative, we have $\epsilon(v)=-1$, otherwise $\epsilon(v)=1$. Finally, $\left.\phi_{f} \equiv \phi\right|_{f}$ are the respective restrictions of the embedding map. The above form of the WZ amplitude defines the holonomy $\operatorname{Hol}_{\mathcal{G}_{H}}(\phi)$ of the bundle gerbe $\mathcal{G}_{H}$ over the world-sheet. The fundamental rôle of the amplitude in the (quantised) theory follows from the fact that it enters the path-integral definition of correlations functions of local operators $\widehat{\mathcal{O}}_{i}$ as

$$
\left\langle\prod_{I} \widehat{\mathcal{O}}_{I}\right\rangle \equiv \int \mathscr{D} \phi \mathrm{e}^{-\mathcal{S}_{k i n}[\phi]} \cdot \mathrm{e}^{-\mathrm{i} \int_{\Sigma} \phi^{*} \mathrm{~d}^{-1} H} \cdot \prod_{I} \mathcal{O}_{I}(\phi) .
$$

\subsection{Twisted gauge fields on $\mathcal{G}_{H}$-branes}

Now that we have acquainted ourselves with the closed-string case, let us consider a boundary CFT describing open strings, defined on a world-sheet whose boundary $\partial \Sigma=\bigsqcup_{\alpha} S_{\alpha} \neq$ $\emptyset$ may have several connected components $S_{\alpha}$. In this setting, the requirement of the unambiguity of the WZ amplitude necessitates the introduction of extra geometric structure - a set of gerbe modules $\mathcal{E}_{\alpha} \longmapsto \mathscr{D}_{\alpha}$, also termed twisted gauge bundles [38], one for each of the $\mathcal{G}_{H}$-brane submanifolds $\mathscr{D}_{\alpha} \supset \phi\left(S_{\alpha}\right)$ in which the corresponding boundary components $S_{\alpha}$ are embedded by $\left.\phi\right|_{S_{\alpha}} \equiv \phi_{\alpha}$. A correction to the bulk expression (3.2) for 
the WZ amplitude given by the product of their holonomies along the respective boundary components of the world-sheet renders the amplitude unambiguous by removing its dependence on the choice of local data for the bundle gerbe $\mathcal{G}_{H}$ in the presence of the world-sheet boundary. The latter property of the correction follows straightforwardly from the twisted character of the gluing rules

$$
\begin{array}{r}
\left.\left(\mathrm{A}_{j}-\mathrm{G}_{i j}^{-1} \mathrm{~A}_{i} \mathrm{G}_{i j}-\mathrm{iG}_{i j}^{-1} \mathrm{dG}_{i j}+\alpha_{i j} \otimes \mathbf{1}_{N}\right)\right|_{\mathscr{O}_{i j}}=0, \\
\left.\left(\mathrm{G}_{j k} \mathrm{G}_{i k}^{-1} \mathrm{G}_{i j}-g_{i j k} \otimes \mathbf{1}_{N}\right)\right|_{\mathscr{O}_{i j k}}=0
\end{array}
$$

for the (local) gauge fields $\mathrm{A}_{i}$ of the twisted gauge bundles. Here, these fields have been defined in terms of the local data for (a specific) $\mathcal{E}_{\alpha}$. The data consist of a family of pairs $\left(\mathrm{A}_{i}, \mathrm{G}_{i j}\right) \in \check{C}^{0}\left(\mathscr{O}, \underline{\Omega}^{1} \otimes \mathfrak{u}(N)\right) \oplus \check{C}^{1}(\mathscr{O}, \underline{U(N)})$ associated to a good covering $\mathscr{O} \equiv\left\{\mathscr{O}_{i}\right\}_{i \in \mathcal{I}_{\alpha}}$ of the submanifold $\mathscr{D}_{\alpha}$ (compatible, in the obvious sense, with $\mathscr{U}$ ).

Given a suitable triangulation $\triangle_{S_{\alpha}}=\left\{e_{\alpha}, v_{\alpha}\right\}$ of the connected component $S_{\alpha}$ of $\partial \Sigma=\bigsqcup_{\alpha} S_{\alpha}$, with $e_{\alpha}$ denoting boundary edges and $v_{\alpha}$ the boundary vertices, the holonomy of the corresponding gerbe module $\mathcal{E}_{\alpha}$ over $S_{\alpha}$ can be expressed succinctly as

$$
\operatorname{Hol}_{\mathcal{E}_{\alpha}}\left(\phi_{\alpha}\right)=\operatorname{tr}_{U(N)}\left(P \prod_{e_{\alpha} \in \triangle_{S_{\alpha}}} \mathrm{e}^{\mathrm{i} \int_{e_{\alpha}} \phi_{e_{\alpha}}^{*} \mathrm{~A}_{i_{\alpha}}} \prod_{v_{\alpha} \subset e_{\alpha}} \mathrm{G}_{i_{v_{\alpha}} i_{e_{\alpha}}}^{\epsilon\left(v_{\alpha}\right)}\left(\phi_{\alpha}(v)\right)\right)
$$

where $P$ denotes the standard path ordering. As previously, $\epsilon\left(v_{\alpha}\right)=-1$ for a negatively oriented vertex and $\epsilon\left(v_{\alpha}\right)=1$ otherwise. The above holonomy completes the definition of the WZ amplitude in the boundary case as per

$$
\mathrm{e}^{\mathrm{i} \int_{\Sigma} \phi^{*} \mathrm{~d}^{-1} H} \equiv \operatorname{Hol}_{\mathcal{G}_{H}}(\phi) \cdot \prod_{\alpha} \operatorname{Hol}_{\mathcal{E}_{\alpha}}\left(\phi_{\alpha}\right)
$$

which is now free of ambiguities.

The central distinguishing feature of the twisted gauge field is its dependence on the gerbe data, as reflected in the transformation rules (3.4). The torsion potential is seen to exchange, via the $\alpha_{i j}$-terms, some of its degrees of freedom with the gauge field. This leads us to consider non-trivial transformations of $\mathrm{A}_{i}$ under tangent-space translations,

$$
\mathrm{A}_{i} \longmapsto \mathrm{A}_{i}+\widetilde{\Pi}_{i} \otimes \mathbf{1}_{N}
$$

with $\widetilde{\Pi}_{i}$ defining, as previously, the gauge shift of the torsion potential (curving) $B_{i}$. In this way, we uncover a scalar-shift extension of the standard gauge symmetry as a direct consequence of the twisted nature of the gauge fields supported by $\mathcal{G}_{H}$-branes. The presence of this extension compels us to consider two possible definitions of the field-strength tensor - the "standard" one, $\mathrm{F}_{i}=\mathrm{dA}_{i}+\mathrm{i}_{i}^{2}$, with twisted gluing/gauge-transformation properties

$$
\left.\left(\mathrm{F}_{j}-\mathrm{G}_{i j}^{-1} \mathrm{~F}_{i} \mathrm{G}_{i j}+\mathrm{d} \alpha_{i j} \otimes \mathbf{1}_{N}\right)\right|_{\mathscr{O}_{i j}}=0, \quad \mathrm{~F}_{i} \longmapsto \mathrm{H}_{i}^{-1} \mathrm{~F}_{i} \mathrm{H}_{i}+\mathrm{d} \widetilde{\Pi}_{i} \otimes \mathbf{1}_{N},
$$

alongside a twisted one, $\mathcal{F}_{i}=\mathrm{F}_{i}+B_{i} \otimes \mathbf{1}_{N}$, with the "standard" gluing/gauge-transformation properties

$$
\left.\left(\mathcal{F}_{j}-\mathrm{G}_{i j}^{-1} \mathcal{F}_{i} \mathrm{G}_{i j}\right)\right|_{\mathscr{O}_{i j}}=0, \quad \mathcal{F}_{i} \longmapsto \mathrm{H}_{i}^{-1} \mathcal{F}_{i} \mathrm{H}_{i}
$$


(above, $\mathrm{H}_{i} \in U(N)$ define the standard (unitary) component of a gauge transformation). The latter is the familiar combination of the gauge field and the Kalb-Ramond background appearing, in particular, in the effective Born-Infeld Lagrangian invariant under the shift $(\mathrm{F}, B) \longmapsto(\mathrm{F}+\mathrm{d} \widetilde{\Pi}, B-\mathrm{d} \widetilde{\Pi})$.

We remark in passing that in view of the fact that the distinguished gerbe $\mathscr{G}_{H}$ is prone to arise in any torsion geometry it would be interesting to study the physical nature of the lower-rank components of the local torsion gerbe data, i.e. the connection $\left(\alpha_{i j}\right)_{i, j \in \mathcal{I}}$ and the transition functions $\left(g_{i j k}\right)_{i, j, k \in \mathcal{I}}$, from the point of view of the theory of gravity (to our knowledge, no such study has been performed so far).

In the case of our immediate interest, i.e. for $\mathcal{M}=G$ a compact simple and simply connected Lie group, and for $\mathscr{D}_{\alpha}$ given by the conjugacy classes

$$
\mathcal{C}_{\lambda}=\left\{h \mathrm{e}^{\frac{2 \pi i \lambda}{k}} h^{-1} \quad \mid h \in G\right\}, \quad \lambda \in P_{+}^{\mathrm{k}}(\mathfrak{g})
$$

of $G$ wrapped by stable (untwisted) maximally symmetric WZW D-branes (or their onesided $G$-translates), the gauge group of each $\mathcal{E}_{\alpha}$ could a priori be arbitrary, as long as it contains a distinguished $U(1)$ subgroup. Indeed, it was shown in [29, 30] that the gauge bundle reduces to a (direct sum) of twisted $U(1)$ gauge bundles for any (stack of) untwisted maximally symmetric D-brane(s) on a simply connected Lie-group manifold $G$. For $\mathscr{D}_{\alpha} \equiv \mathcal{C}_{\lambda_{\alpha}}$ as above, the field strength of the corresponding twisted gauge field is given by

$$
\mathcal{F}\left(h \mathrm{e}^{\frac{2 \pi i \lambda_{\alpha}}{\mathrm{k}}} h^{-1}\right)=\frac{\mathrm{k}}{4 \pi} \operatorname{tr}\left[\left(h^{-1} \mathrm{~d} h\right) \mathrm{e}^{\frac{2 \pi i \lambda_{\alpha}}{\mathrm{k}}}\left(h^{-1} \mathrm{~d} h\right) \mathrm{e}^{-\frac{2 \pi i \lambda_{\alpha}}{\mathrm{k}}}\right] \otimes \mathbf{1}_{N}
$$

and is globally defined and Abelian. Granted the existence of a $U(1)$ component of the gauge group of the twisted gauge bundle, we may readily convince ourselves that the twisted gauge field splits into a flat untwisted (possibly) non-Abelian component and a twisted $U(1)$ component with the curvature defining $\mathscr{D}_{\alpha}$ as above.

From our discussion so far, there emerges a dual interpretation of the Kalb-Ramond field $B$ - on the one hand (Sect.2.3), we identify it as a torsion potential, that is a gauge field for the tangent-space translational symmetry (of the $\sigma$-model), with the simple transformation property (2.13) under tangential coordinate shifts; on the other hand (Sect. 3.2), it reappears as part of a rich differential-geometric structure of a bundle gerbe $\mathcal{G}_{H} \underset{\text { loc. }}{\longrightarrow}(B, \alpha, g)$ with the globally defined (closed) 3 -form field $H$ as its curvature, induced in the target manifold of the $\sigma$-model due to the presence of the topological WZ term. Accordingly, the equivalence relations (3.1), expressing the notion of a stable isomorphism of bundle gerbes in the local language, acquire the geometric interpretation of gauge equivalences for the symmetry group of tangent-space translations. The latter symmetry is in turn transmitted through the scalar twist term into the $U(1)$-reducible twisted gauge theory supported by a $\mathcal{G}_{H}$-brane world-volume and thus augments the standard gauge symmetry. In fact, this scalar-shift extension of the unitarily implemented gauge symmetry can be thought of as the lowest-order term in the $\ell^{-1}$ expansion of the full translational symmetry explored in 18 . 
It should be remarked that there arises an elementary discrepancy between the situation described here and the one encountered in the non-commutative matrix geometry: in the smooth ( $\sigma$-model) setting, the gauge field is defined as tangential to the $\mathcal{G}_{H}$-brane world-volume, and by construction one allows only those gauge transformations of the gerbe which keep the world-sheet boundary fixed. In what follows, we shall ultimately relax this constraint in order to account for shape fluctuations of $\mathcal{G}_{H}$-brane world-volumes, in a manner consistent with our geometric interpretation of the gauge freedom underlying the structure of the torsion gerbe.

\section{The spectral geometry of the BCFT}

We now turn to studying the low-energy effective action (1.3) within the framework of noncommutative geometry. That the world-volumes of maximally symmetric WZW branes can be viewed as quantisations of conjugacy classes in the group target was already shown in [1], and the action (1.3) indeed has the form of a gauge theory on such a quantised conjugacy class - taking, however, a very special form. In this section, we will first introduce the main ingredients of the non-commutative spaces that come into play (more details are spelled out in the Appendix) and then formulate an extended gauge principle - borrowed from the classical gerbe picture of WZW branes - which singles out the action (1.3) among a large class of actions that enjoy "conventional" gauge invariance. On the way, we will also remark that Weitzenböck geometry appears naturally on non-commutative WZW branes as well.

\subsection{The spectral data}

We first show how to extract a spectral triple from boundary WZW models. The construction is inspired by Fröhlich's and Gawędzki's work [39] on the non-commutative geometry of closed strings, but adapted to the boundary case - which in fact affords more natural choices of algebras and Hilbert spaces than the bulk setting.

Following Connes [40, the starting point of studying a non-commutative space is its spectral triple consisting of an algebra $\mathcal{A}$ (generalising the algebra of suitable functions on a manifold), a generalised Dirac operator $D$, and a Hilbert space $\mathcal{H}$ (generalising the space of square-integrable spinors) on which both act. Quantum field theories come with algebras and Hilbert spaces in any case, and in conformal QFT there is also a natural candidate, namely the Virasoro mode $L_{0}$, for a generalised Laplace operator (see in particular 41] for work exploiting this); in superconformal field theories, there is an equally natural candidate for the Dirac operator, namely the mode $G_{0}$ of the superconformal current - acting on the Ramond sector of the state space.

In the Appendix, we describe the structure of supersymmetric WZW models in some detail (following [42]), here we merely list the ingredients of the non-commutative spectral data associated to those SCFTs on world-sheets with boundary. Note that passing to the supersymmetric version does not affect the structure of WZW models significantly: the bosonic sector stays the same except for the shifting of the level by the dual Coxeter number of the group, and is tensored by decoupled free fermions. 
We start from a boundary WZW model with a maximally symmetric boundary condition labelled by $\lambda \in P_{+}^{\mathrm{k}}(\mathfrak{g})$. As we are interested in the low-energy effective action, we restrict to the subspace $\mathcal{H}_{\mathrm{NS}}^{\lambda}$ of states (from the NS sector) whose conformal dimensions tend to zero in the limit (1.5), i.e. to NS-primaries and their descendants under the finite-dimensional horizontal Lie (sub)algebra $\left[\widehat{\mathfrak{g}}_{\mathrm{k}}\right]_{(0)} \cong \mathfrak{g}$. We take the boundary fields associated to those states as our algebra of "functions" $\mathcal{A}_{\lambda}$, with multiplication induced by the Operator Product Expansion (OPE) in the decoupling limit (where all singularities disappear and where the product becomes associative) - see [1]. This algebra is in fact a full matrix algebra of size equal to the dimension of the irreducible representation of $\mathfrak{g}$ labelled by $\lambda$; extension to stacks of $n$ identical D-branes is trivial.

This algebra acts on the whole state space of the theory, but we restrict to the Rsector - as we also want the "Dirac operator" $G_{0}$ to act - and to the states of the lowest conformal dimension within that; we call this subspace $\mathcal{H}^{\lambda}$. The spectral triple associated to our model is therefore

$$
\mathcal{T}_{\text {WZW }}^{\lambda}:=\left(\mathcal{A}^{\lambda}, D_{\mathrm{R}}^{\lambda}, \mathcal{H}^{\lambda}\right)
$$

The action of the algebra on the Hilbert space is compatible with its OPE-induced product. The explicit formula for the Dirac operator can be derived from expanding $G_{0}$ in terms of fermion and current modes (see the Appendix); it reads

$$
D_{\mathrm{R}}^{\lambda}:=-\left.\mathrm{i} \sqrt{\mathrm{k}} G_{0}\right|_{\mathcal{H}^{\lambda}}=\gamma^{a} \otimes L_{a}^{\lambda}-\frac{\mathrm{i}}{12} f_{a b c} \gamma^{a} \gamma^{b} \gamma^{c} \otimes \mathbf{1}_{D_{\lambda}},
$$

where the $\gamma^{a}$ are rescaled fermion modes (satisfying the Clifford algebra), and the tensor product is with respect to the above-mentioned factorisation of the supersymmetric WZW model into a bosonic part and free fermions. $L_{a}^{\lambda}$ denotes the action of horizontal-subalgebra generators $\widehat{J}_{0}^{a}$ on the space $\mathcal{H}_{\mathrm{NS}}^{\lambda}$ of dimension $D_{\lambda}$,

$$
L_{a}^{\lambda} \triangleright f \equiv\left[Y_{a}^{\lambda}, f\right]:=\widehat{J}_{0}^{a} f
$$

for all $f \in \mathcal{H}_{\mathrm{NS}}^{\lambda}$, where $Y_{a}^{\lambda}$ denote the standard linear combinations of the vector hyperharmonics of $\mathfrak{g}$ compatible with the choice of the Lie-algebra basis.

In summary, our choice of spectral data leads to a finite-dimensional matrix geometry for the quantised brane world-volume. It seems to be the only natural choice from the point of view of low-energy effective string theory: focussing on low-energy modes dictates the truncation, open-string couplings via OPE induce the algebra structure.

It is relatively straightforward to derive the spaces of differential forms $\widetilde{\Omega}_{D_{\mathrm{R}}^{\lambda}}^{p}\left(\mathcal{A}^{\lambda}\right)$ and the action of the exterior differential $d$ from the spectral triples $\mathcal{T}_{\text {WZW }}^{\lambda}$, following Connes' general construction; a similar computation has been performed, in the closedstring setting, in [43, 44]. Details are given in the Appendix; here, let us just recall that on a $p$-form

$$
\omega=\sum_{i \in I} a_{i}^{(0)}\left[D_{\mathrm{R}}^{\lambda}, a_{i}^{(1)}\right]\left[D_{\mathrm{R}}^{\lambda}, a_{i}^{(2)}\right] \cdots\left[D_{\mathrm{R}}^{\lambda}, a_{i}^{(p)}\right], \quad a_{i}^{(n)} \in \mathcal{A}^{\lambda},
$$

the exterior differential acts as

$$
\mathrm{d} \omega=\sum_{i \in I}\left[D_{\mathrm{R}}^{\lambda}, a_{i}^{(0)}\right]\left[D_{\mathrm{R}}^{\lambda}, a_{i}^{(1)}\right]\left[D_{\mathrm{R}}^{\lambda}, a_{i}^{(2)}\right] \cdots\left[D_{\mathrm{R}}^{\lambda}, a_{i}^{(p)}\right] .
$$


Due to the underlying group symmetry and to simplifications characteristic for matrix geometries [45, 46], the spaces of forms can be written rather neatly as

$$
\widetilde{\Omega}_{D_{\mathrm{R}}^{\lambda}}^{p}\left(\mathcal{A}^{\lambda}\right) \cong\left\{\gamma^{a_{1} a_{2} \ldots a_{p}} \otimes f_{a_{1} a_{2} \ldots a_{p}} \quad \mid \quad f_{a_{1} a_{2} \ldots a_{p}} \in \mathcal{A}^{\lambda}\right\}
$$

where the $\gamma^{a_{1} a_{2} \ldots a_{p}}$ are totally antisymmetric products of $\gamma^{a}$.

It is worth noting that the differential calculus thus constructed is naturally $d$-dimensional. In D-brane language, this means that it does not allow for a gauge-covariant split of the gauge-field 1-forms $A$ into components tangent to the world-volume $\mathcal{C}_{\lambda}$ (this would represent a "bona fide" gauge field) and those normal to it (representing transverse scalars).

Our finite-dimensional calculus allows to define a Hodge star operator $\star_{H}$, mapping $p$-forms to $(d-p)$-forms: As shown in [45], the Hodge operator can be chosen in precisely the same form as in the commutative case; with our flat metric $\delta_{a b}$, the Hodge star $\star_{H}$ simply amounts to contracting form indices with the totally antisymmetric tensor of rank $d$.

We will need differential forms in our study of gauge-invariant actions on the quantised world-volume below. Before turning to that, let us pause to characterise the geometry associated to the spectral data $\mathcal{T}_{\text {WZW }}^{\lambda}$ further. On the free $\mathcal{A}^{\lambda}$-module $\widetilde{\Omega}_{D_{\mathrm{R}}^{\lambda}}^{1}$, there is a natural definition of a connection [39], namely

$$
\nabla_{\mathrm{R}}^{\lambda}: \widetilde{\Omega}_{D_{\mathrm{R}}^{\lambda}}^{1}\left(\mathcal{A}^{\lambda}\right) \ni \omega \longmapsto \nabla_{\mathrm{R}}^{\lambda} \omega:=\gamma^{a} \otimes\left(\mathrm{i} L_{a}^{\lambda} \triangleright \omega\right) \in \widetilde{\Omega}_{D_{\mathrm{R}}^{\lambda}}^{1}\left(\mathcal{A}^{\lambda}\right) \otimes_{\mathcal{A}^{\lambda}} \widetilde{\Omega}_{D_{\mathrm{R}}^{\lambda}}^{1}\left(\mathcal{A}^{\lambda}\right)
$$

As was first shown in the closed-string setting in [39], this connection has two fundamental properties: First, it parallelises the fuzzy geometry at hand,

$$
\nabla_{\mathrm{R}}^{\lambda}\left(\gamma^{a} \otimes \mathbf{1}_{\operatorname{dim} \mathcal{H}_{\mathrm{NS}}^{\lambda}}\right)=0
$$

and hence has a vanishing curvature,

$$
\mathcal{R}\left(\nabla_{\mathrm{R}}^{\lambda}\right) \equiv-\left.\left(\nabla_{\mathrm{R}}^{\lambda}\right)^{2}\right|_{\widetilde{\Omega}_{D_{\mathrm{R}}^{\lambda}}^{1}\left(\mathcal{A}^{\lambda}\right)}=0 .
$$

Secondly, it has a non-vanishing torsion

$\mathcal{T}\left(\nabla_{\mathrm{R}}^{\lambda}\right)\left(\gamma^{a} \otimes \mathbf{1}_{\operatorname{dim} \mathcal{H}_{\mathrm{NS}}^{\lambda}}\right) \equiv\left[\left(\mathrm{d}-m \circ \nabla_{\mathrm{R}}^{\lambda}\right)\left(\gamma^{a} \otimes \mathbf{1}_{\operatorname{dim} \mathcal{H}_{\mathrm{NS}}^{\lambda}}\right)\right]_{2 \text { form }}=\frac{1}{2} f_{a b c} \gamma^{b c} \otimes \mathbf{1}_{\operatorname{dim} \mathcal{H}_{\mathrm{NS}}^{\lambda}}$,

where $m: \widetilde{\Omega}_{D_{\mathrm{R}}^{\lambda}}^{1}\left(\mathcal{A}^{\lambda}\right) \otimes_{\mathcal{A}^{\lambda}} \widetilde{\Omega}_{D_{\mathrm{R}}^{\lambda}}^{1}\left(\mathcal{A}^{\lambda}\right) \longmapsto \widetilde{\Omega}_{D_{\mathrm{R}}^{\lambda}}^{2}\left(\mathcal{A}^{\lambda}\right)$ is the natural projection map (which eliminates the "junk forms", see the Appendix). In other words, we find the characteristic properties of a (non-commutative) Weitzenböck geometry! We are led to conclude that the effective fuzzy D-brane geometry inherits crucial features of its smooth, classical counterpart, where the Cartan-Killing metric and the Kalb-Ramond 3-form conspire in such a way that the corresponding connection is flat and torsion-full. The associated notion of teleparallelism, with its principle of gauging tangential translations, will be invoked and exploited in the next subsection. 


\subsection{Non-commutative gauge symmetries: ordinary and extended}

Having reconstructed the differential calculus from the spectral triple $\mathcal{T}_{\text {WZW }}^{\lambda}$, we may next consider the most natural gauge dynamics based on it: We build a field-strength 2-form

$$
F(A)=[\mathrm{d} A+A \wedge A]_{2 \text {-form }}
$$

(the subscript refers to projecting out "junk forms"), and use this to obtain the (noncommutative) Yang-Mills action functional

$$
\mathcal{S}_{\mathrm{YM}}[A] \equiv \frac{1}{4 g_{\mathrm{YM}}^{2}} \operatorname{Tr}_{\mathcal{H}^{\lambda}}\left[F(A) \wedge \star_{H} F(A)\right]
$$

where $\operatorname{Tr}_{\mathcal{H}^{\lambda}} \equiv \frac{2}{D} \operatorname{tr}_{\Omega_{\mathrm{R}}} \otimes \operatorname{tr}_{\mathcal{H}_{\mathrm{NS}}^{\lambda}}$, with $D$ the dimension of the Clifford module, and $g_{\mathrm{YM}}$ is the coupling constant of the gauge theory. The Clifford algebra (A.8) satisfied by $\gamma^{a}$ then enables us to rewrite the above expression in the standard index notation

$$
\mathcal{S}_{\mathrm{YM}}[A]=\frac{1}{4 g_{\mathrm{YM}}^{2}} \operatorname{tr}_{\mathcal{H}_{\mathrm{NS}}^{\lambda}}\left[F_{a b}(A) F_{a b}(A)\right]
$$

where - by virtue of (4.2) - the field strength takes the form familiar from (1.4), see the Appendix for some details.

Since $F_{a b}$ contains a contribution $f_{a b c} A_{c}$, the Yang-Mills action (4.3) actually has a mass term for the gauge field. Nevertheless, it is the simplest and most natural action invariant under the action of the gauge group [40]

$$
\mathcal{U}\left(\mathcal{A}^{\lambda}\right):=\left\{u \in \mathcal{A}^{\lambda} \quad \mid u u^{*}=\mathbf{1}_{D_{\lambda}}=u^{*} u\right\} \cong U\left(D_{\lambda}\right)
$$

under which the gauge field transforms as usual

$$
A \longmapsto A^{u}:=u^{*} A u+\mathrm{i} u^{*} \mathrm{~d} u .
$$

Thus far, everything is standard in non-commutative geometry. One might, however, notice that, due to the defining identity for torsion,

$$
\mathrm{d} \equiv m \circ \nabla_{\mathrm{R}}^{\lambda}+\mathcal{T}\left(\nabla_{\mathrm{R}}^{\lambda}\right)
$$

the Yang-Mills action (4.3) can be viewed as a model of a gauge field $A$ in a (noncommutative) Weitzenböck geometry, which is coupled "minimally" to the torsion field" This observation, taken together with the identification of the gauge field $A$ as an excitation of the twisted background (3.7), suggests to look for a non-commutative implementation of gerbe structures, in particular of the translational symmetry. To this end, we propose to consider the non-commutative analogue

$$
A^{u, \Pi}=A^{u}+\Pi
$$

of the classical transformation behaviour (3.5) of the gauge field. (In view of the decomposition $X_{a}=Y_{a}+A_{a}$, the non-commutative gauge field $A_{a}$ scales like a covariant

\footnotetext{
${ }^{8}$ Similar couplings have been indeed considered in classical teleparallel gravity, cf. 47.
} 
coordinate, while the classical gauge field from (3.5) scales like a covariant derivative; the relation between the two has been worked out for flat backgrounds in [61] and involves the $B$-field in just the same way as the passage from $\Pi$ to $\widetilde{\Pi}$ in (2.12). This explains why in (4.4) we see $\Pi$ rather than $\widetilde{\Pi}$.)

We restrict the 1 -form $\Pi$ to be of the form

$$
\Pi=\gamma^{a} \otimes \pi_{a} \cdot \mathbf{1}_{D_{\lambda}}, \quad \text { with } \quad \pi_{a} \in \mathbb{C},
$$

which is necessary and sufficient for the two symmetries (unitary gauge transformations and scalar shifts) to commute with one another. This is in turn motivated by the results of Sect.2.3 in which we identified world-sheet-constant tangential translations as a remnant of left-translations in the group, independent of the more familiar adjoint group action ${ }^{9}$. Thus, our extended gauge symmetry group is $\mathcal{U}\left(\mathcal{A}^{\lambda}\right) \times \mathbb{C}^{d}$. Eq. (4.4) implies the simple transformation behaviour

$$
F^{u, \Pi}=F^{u}+\mathrm{d} \Pi, \quad F^{u}=u^{*} F u
$$

for the gauge-field strength under the extended symmetry - compare to (3.6) and (2.12) in the bundle-gerbe context.

Note that in the case of flat D-branes carrying a constant Kalb-Ramond field (with $H=0$ ), one has $\mathrm{d} \Pi=0$ so that $F$ is invariant under shifts; our extension of the unitary symmetry is non-trivial only for curved backgrounds. This is well in keeping with the observation that the Yang-Mills term of the matrix model for flat D-branes does not receive any corrections, to leading order of the perturbative expansion.

In the WZW case, on the other hand, postulating invariance under extended gauge transformations allows us to derive non-trivial corrections to the Yang-Mills action. Thus, we introduce the corrected action

$$
\mathcal{S}_{\mathcal{E}}[A]=\mathcal{S}_{\mathrm{YM}}[A]+\Delta_{\mathcal{E}} \mathcal{S}[A]
$$

and demand the relation

$$
\mathcal{S}_{\mathrm{YM}}\left[A^{u, \Pi}\right]+\Delta_{\mathcal{E}} \mathcal{S}\left[A^{u, \Pi}\right] \stackrel{!}{=} \mathcal{S}_{\mathrm{YM}}[A]+\Delta_{\mathcal{E}} \mathcal{S}[A]
$$

to hold for $A^{u, \Pi}$ as above. Among admissible extensions $\Delta_{\mathcal{E}} \mathcal{S}$ of the non-commutative Yang-Mills functional $\mathcal{S}_{\mathrm{YM}}$ for untwisted gauge fields, there is a distinguished class - the minimal ones, with at most two derivatives of the gauge field. In what follows, we restrict our search to such minimal extensions.

Any extension satisfying (4.5) should in particular provide, upon a scalar variation $A \longmapsto A+\Pi$, a counterterm for the part of the variation of the Yang-Mills functional that is quadratic in $\Pi$,

$$
\left(\delta_{\Pi} \mathcal{S}_{\mathrm{YM}}[A]\right)_{\pi^{2}}=\frac{g^{\vee} N}{2 g_{\mathrm{YM}}^{2}} \delta^{a b} \pi_{a} \pi_{b} .
$$

In the present case, the most systematic method of constructing a suitable extension of the Yang-Mills action is to write down all possible expressions quadratic in $A$ and then

\footnotetext{
${ }^{9}$ But see comments at the bottom of p. 14 .
} 
isolate those that both have the required index structure (4.6) and admit a completion (by higher-order terms in $A$ ) that makes them invariant under standard unitary gauge transformations. Listing all possible terms is much easier in a covariant notation employing the exterior derivative, the wedge product and the Hodge star. In this notation, the variation in (4.6) is proportional to $\Pi \wedge \star_{H} \Pi$.

We arrive at twelve distinct candidates ${ }^{10}$ for the quadratic counterterm in the extension

$$
\begin{gathered}
A \wedge A, \quad A \wedge \star_{H} A, \\
A \wedge \mathrm{d} A, \quad A \wedge \star_{H} \mathrm{~d} A, \quad \star_{H} A \wedge \star_{H} \mathrm{~d} A, \\
A \wedge \star_{H} \mathrm{~d} \star_{H} A, \quad \star_{H} A \wedge \star_{H} \mathrm{~d} \star_{H} A, \\
\mathrm{~d} A \wedge \mathrm{d} A, \quad \mathrm{~d} A \wedge \star_{H} \mathrm{~d} A, \\
\mathrm{~d} A \wedge \star_{H} \mathrm{~d} \star_{H} A, \quad \star_{H} \mathrm{~d} A \wedge \star_{H} \mathrm{~d} \star_{H} A, \quad \star_{H} \mathrm{~d} \star_{H} A \wedge \star_{H} \mathrm{~d} \star_{H} A .
\end{gathered}
$$

Note that we do not demand the forms to be of top degree $d$ since in our matrix geometry the integral is replaced by the trace. Therefore, the form degree is not directly relevant, as long as it does not exceed $d$ (which is why $\star_{H} A \wedge \star_{H} A$ does not appear in the list: this form would have degree $2 d-2>d$ ).

We can exclude all but one of the above candidates: First, note that the five terms containing the divergence $\star_{H} \mathrm{~d} \star_{H} A$ differ in their index structure from (4.6) (written out, the divergence of $A$ reads $L_{a} A_{a}$ ), and therefore they cannot cancel that variation.

Likewise, the expressions $A \wedge A, A \wedge \star_{H} \mathrm{~d} A$ and $\mathrm{d} A \wedge \mathrm{d} A$ have the wrong index structures (e.g., $A \wedge A \sim \varepsilon^{a b} A_{a} A_{b}$ ) and cannot be used to cancel (4.6) - even though the last of these three terms admits the gauge invariant completion $F \wedge F$.

The expression $\mathrm{d} A \wedge \star_{H} \mathrm{~d} A$ can be dropped as its completion is just the original YangMills term $F \wedge \star_{H} F$.

For dimensional reasons, the term $\star_{H} A \wedge \star_{H} \mathrm{~d} A$ exists only for $d \leq 3$, i.e. on $S U(2)$, so it is not a universal extension (actually, being a top form on $S U(2)$, it reduces to $A \wedge \mathrm{d} A$ in this case).

Of the remaining two candidates, $A \wedge \star_{H} A$ is the standard mass term for the gauge field and shall consequently be dropped, too, despite its right index structure: again, it has no gauge invariant completion.

The last remaining term $A \wedge \mathrm{d} A$ does have the right structure and can be completed to a gauge invariant Chern-Simons current ${ }^{11}$

$$
K(A)=A \wedge \mathrm{d} A+\frac{2}{3} A \wedge A \wedge A \equiv\left[A\left\{D_{\mathrm{R}}^{\lambda}, A\right\}+\frac{2}{3} A^{3}\right]_{3 \text {-form }} \equiv \gamma^{a b c} \otimes C S_{a b c} .
$$

\footnotetext{
${ }^{10}$ One should keep in mind that they all appear under the trace, which implies certain obvious equivalences that we have taken into account.

${ }^{11}$ We use the symbol $\gamma^{a b c} \equiv \frac{1}{3 !} \sum_{\sigma \in \mathfrak{S}_{3}} \operatorname{sign}(\sigma) \gamma^{\sigma(a)} \gamma^{\sigma(b)} \gamma^{\sigma(c)}$.
} 
Thus, after the dust has cleared, we are left with the unique minimal extension which reads

$$
\Delta_{\mathcal{E}} \mathcal{S}[A] \equiv \mathcal{S}_{C S}[A]=\frac{1}{g_{\mathrm{YM}}^{2}} \operatorname{Tr}_{\mathcal{H}^{\lambda}}\left[T\left(\nabla_{\mathrm{R}}^{\lambda}\right) \wedge \star_{H} K(A)\right]
$$

with the fuzzy torsion 3-form

$$
T\left(\nabla_{\mathrm{R}}^{\lambda}\right):=\frac{\mathrm{i}}{3 !} f_{a b c} \gamma^{a b c} \otimes \mathbf{1}_{N}
$$

This shows that our former interpretation of the Yang-Mills term as a model of a gauge field coupled minimally to the torsion field just as well applies to the Chern-Simons extension.

As mentioned in the introduction already, it is straightforward to check its standard unitary gauge invariance. And invariance under the scalar shift $A \longmapsto A+\Pi$ to all orders in $\Pi$, not just quadratic, was also observed before.

Altogether, for a suitable choice of the coupling constant $g_{\mathrm{YM}}$, the complete model that we obtain is just our original matrix model action,

$$
\mathcal{S}_{\mathcal{E}}[A]=\frac{1}{4 g_{\mathrm{YM}}^{2}} \operatorname{Tr}_{\mathcal{H}^{\lambda}}\left[F(A) \wedge \star_{H} F(A)\right]+\frac{1}{g_{\mathrm{YM}}^{2}} \operatorname{Tr}_{\mathcal{H}^{\lambda}}\left[T\left(\nabla_{\mathrm{R}}^{\lambda}\right) \wedge \star_{H} K(A)\right] \equiv \mathcal{S}_{\mathrm{ARS}}[A] .
$$

\section{Conclusions and outlook}

We have seen that the low-energy effective action (1.3) of open strings in WZW models can be rederived from the basic building blocks of the non-commutative geometry, associated via supersymmetric BCFT to maximally symmetric D-branes, by invoking invariance under extended shift-gauge transformations as a symmetry principle. Then the nonstandard term in the action - the three-dimensional Chern-Simons term with its specific relative coupling - arises necessarily to complement the usual Yang-Mills term.

The spectral triple contains a generalised Dirac operator whose properties (flatness, torsion) in particular suggest a straightforward interpretation of the matrix model as describing the dynamics of a gauge field coupled minimally to the torsion field of a noncommutative Weitzenböck (or teleparallel) geometry.

Torsion is also the source of the extension of the standard principle of gauge symmetry used in that derivation. While standard gauge transformations constitute a unitary implementation of standard target-space isometries preserved by the BCFT, the twisted nature of the gauge field supported by WZW D-brane world-volumes is an immediate consequence of the torsion-gerbe structure present on the WZW manifold. The gauge field of the model is induced by the torsion gerbe and consequently exchanges some of its degrees of freedom with the $B$-field, via its non-trivial transformation properties under the gauge shifts of the Kalb-Ramond torsion potential. We expect this mechanism to play a similar rôle for all $\sigma$-models with curved targets.

We have also shown that the low-energy effective action can be obtained via the canonical quantisation of the linearised version of the underlying WZW $\sigma$-model rewritten in the Schild gauge, based on a generic Poisson structure on the (Euclidean) world-sheet.

The derivation naturally identifies D0-branes as the elementary degrees of freedom of the matrix model - an interpretation which conforms with the world-sheet RG analysis, showing that higher-dimensional branes can be viewed as bound states of D0-branes. 
That it is at all possible to derive the low-energy effective action for open strings in group manifolds from the linearised (bulk) $\sigma$-model action remains mysterious to us. One might argue that, after all, WZW models are relatively close to free theories, but we feel that the results of Sect.2 should hold in greater generality, and that there is some deeper underlying principle to be uncovered. In the flat case, the transition from the Schild action to a matrix model leads to the IKKT model and was argued [4 to provide a simple implementation of the universal stringy uncertainty relation. At the technical level, the rederivation of the fuzzy matrix model from the linearised WZW action is largely reminiscent ${ }^{12}$ of the methods employed in the computation of effective actions for non-linear $\sigma$-models [48] in terms of Riemann normal coordinates (see also [49, 50] and - in a more general context - [51, 52, 53]), with world-sheet quantisation playing the rôle of a natural regularisation procedure. The results of Sect. 2 certainly call for further investigation and clarification of the physics behind them.

A more profound understanding of the structures described in the present paper is achieved in [18] where in particular the extended gauge symmetry is studied in the socalled geometric framework of gerbe theory [34, 35]. This allows - from first principles - to identify the shift symmetry (3.5) as the first-order term (in $\ell$, or $\alpha^{\prime}$ ) of a complete expression. In addition, it affords a generalisation of our results to the case of twisted maximally symmetric D-branes.

There are a number of more concrete open questions to study. An obvious idea is to use the complete symmetry from [18] to determine which higher-order corrections (in $\alpha^{\prime}$ and the gauge field) to the matrix model are allowed; the result should then be compared to higher-order terms in the effective action obtained from boundary CFT. The latter have not been calculated as yet, but the computations of [22, 23] should be useful here. Another interesting issue is that of lifting the gerbe symmetry discussed in our paper to the strictly non-classical régime, and in particular to the $\mathcal{U}_{q}(\mathfrak{g})$-related ${ }^{13}$ geometries of $^{-}$ [54, 55] - one would hope that our findings shed some light on the still unresolved problem of defining a $\mathcal{U}_{q}(\mathfrak{g})$-covariant gauge field theory capturing the dynamics of quantum WZW branes beyond the semiclassical approximation of the fuzzy matrix model. It should also be worthwhile to combine our findings with the investigations in [56, 57] and to see what rôle torsion geometry plays in continuum limits of the matrix model.

Extension beyond models of the WZW type considered may well be tractable: First of all, the equivalence between the Polyakov action and the Schild action can be established for arbitrary (dilaton-free) non-linear $\sigma$-models [18] and hence allows to associate matrix models to any such background. Cosets are a particularly important class, from the point of view of string theory. In this context, it should be noted that the Dirac operator (4.1) is a special case of a more general construct known as Kostant's cubic Dirac operator [58], see also [59], which was studied extensively in the mathematical literature. The general form of this operator given in [58] coincides with the Ramond-Dirac operator derived in [39] for supersymmetric cosets of WZW models. Thus, there is a natural starting point of

\footnotetext{
${ }^{12}$ We thank K. Gawȩdzki for drawing our attention to this analogy.

${ }^{13}$ Here $\mathcal{U}_{q}(\mathfrak{g})$ denotes the Drinfel'd-Jimbo algebra with the deformation parameter $q=\mathrm{e}^{\frac{\pi \mathrm{i}}{\mathrm{k}+g^{\nabla}}}$, as suggested by the quantum symmetries of the (B)CFT structures.
} 
a discussion of effective D-brane geometry and gauge dynamics in the NCG framework for coset $\sigma$-models. We hope to return to this question in the near future.

Scalar shifts of gauge fields such as those appearing in our extended gauge symmetries also occur in the context of Morita self-equivalences of the underlying NCG, see [60, 61, 62, 63, 64, 65]. Thus one might speculate that there is some hidden T-duality group acting on the gerbe data. A related issue is that of the (stable-isomorphism) equivalences among gerbe data: bearing in mind that it is the Kalb-Ramond potential $B$ that defines the non-commutative deformation of the algebra of functions on the D-brane world-volume, it is tempting to relate the ambiguities in its definition (which show up whenever there is a non-vanishing torsion) to some kind of Seiberg-Witten equivalences 61 among various formulations of the gauge field theory on the D-brane, differing in the choice of the noncommutativity parameter. In contrast to the flat case, the kinetic and the topological term in the WZW model action are linked by requiring conformal invariance, so we would at most expect a very restricted set of equivalences.

\section{Acknowledgments}

We are indebted to Krzysztof Gawędzki for numerous helpful discussions and for generously sharing his insights with us. We are also grateful to Jacek Pawełczyk for discussions and his sustained interest in our work.

This work was supported in part by the EU Research Training Network grants 'Euclid', contract number HPRN-CT-2002-00325, 'Superstring Theory', contract number MRTNCT-2004-512194, by the PPARC rolling grant PP/C507145/1, and by the EU Marie Curie Training Site 'Strings, Branes and Boundary Conformal Field Theory', contract number HPMT-CT-2001-00296.

\section{A. Appendix}

In the following, we collect some details on supersymmetric WZW models (a very nice exposition is given in [42] which we largely follow), on spectral data in non-commutative geometry (see [40] and also [43]), and on how to extract the latter from the former.

Our starting point is the supercurrent of the supersymmetric WZW model $(Z:=(z, \theta)$ is the supercoordinate)

$$
\mathscr{T}(Z):=\frac{1}{2} G(z)+\theta T(z)
$$

containing the superconformal current $G$ and the energy momentum tensor $T$ as its superpartner. When written in terms of the super-Kac-Moody current

$$
J^{a}(Z):=\sqrt{2 \mathrm{k}} \psi^{a}(z)+\theta j^{a}(z)
$$

and its superderivative $\left(\mathscr{D}:=\frac{\partial}{\partial \theta}+\theta \partial, \partial=\frac{\partial}{\partial z}\right)$

$$
\mathscr{D} J^{a}(Z)=j^{a}(z)+\theta \sqrt{2 \mathrm{k}} \partial \psi^{a}(z)
$$


the definition reads (the colons denote normal ordering)

$$
\mathscr{T}(Z):=-\frac{1}{\mathrm{k}} \delta_{a b}: \mathscr{D} J^{a} J^{b}:(Z)+\frac{2}{3 \mathrm{k}^{2}} f_{a b c}: J^{a}: J^{b} J^{c}::(Z)+\mathscr{A},
$$

where $\mathscr{A}=0$ in the NS sector, but in the $\mathrm{R}$ sector one has to add the Ramond vacuum energy $\mathscr{A}=\frac{c}{16} Z^{-\frac{3}{2}}$, with $Z^{-\frac{3}{2}}=\theta z^{-2}$.

Above, the $\psi^{a}$ are the fermions of the model, furnishing the adjoint representation of the current-symmetry algebra generated by the currents $j^{a}, a=1,2, \ldots, d$. They satisfy the OPE

$$
\begin{aligned}
j^{a}\left(z_{1}\right) j^{b}\left(z_{2}\right) & \sim-\frac{\mathrm{k} \delta_{a b}}{2\left(z_{1}-z_{2}\right)^{2}}+\frac{f_{a b c}}{z_{1}-z_{2}} j^{c}\left(z_{2}\right), \\
\psi^{a}\left(z_{1}\right) \psi^{b}\left(z_{2}\right) & \sim \frac{\delta_{a b}}{z_{1}-z_{2}}, \\
j^{a}\left(z_{1}\right) \psi^{b}\left(z_{2}\right) & \sim \frac{f_{a b c}}{z_{1}-z_{2}} \psi^{c}\left(z_{2}\right),
\end{aligned}
$$

which simplifies considerably upon redefining the currents as

$$
j^{a} \longmapsto \widehat{j}^{a}:=j^{a}+\frac{1}{2} f_{a b c}: \psi^{b} \psi^{c}: .
$$

Indeed, for the new currents we obtain

$$
\begin{aligned}
& \widehat{j}^{a}\left(z_{1}\right) \widehat{j}^{b}\left(z_{2}\right) \sim-\frac{\widehat{\mathrm{k}} \delta_{a b}}{2\left(z_{1}-z_{2}\right)^{2}}+\frac{f_{a b c}}{z_{1}-z_{2}} \widehat{j}^{c}\left(z_{2}\right), \\
& \widehat{j}^{a}\left(z_{1}\right) \psi^{b}\left(z_{2}\right) \sim 0,
\end{aligned}
$$

with the shifted value of the level $\widehat{k}:=\mathrm{k}-g^{\vee}$, and the original quantum field theory dissolves into a pair of mutually independent subtheories: a bosonic $\widehat{\mathfrak{g}}_{\widehat{\mathrm{k}}}$ WZW model and the theory of $d$ free Fermi fields $\psi^{a}$.

Upon substituting (A.2) and A.3) in (A.4) and using (A.1) together with (A.6), we arrive at the formula

$$
-\sqrt{2 \mathrm{k}} G(z)=\delta_{a b}: \widehat{j}^{a} \psi^{b}:(z)-\frac{1}{3} f_{a b c}: \psi^{a}: \psi^{b} \psi^{c}::(z) .
$$

In terms of the Laurent modes

$$
G(z)=\sum_{m \in \mathbb{Z}} z^{-m-\frac{3}{2}} G_{m}, \quad \widehat{j}^{a}(z)=\sum_{m \in \mathbb{Z}} z^{-m-1} \widehat{j}_{m}^{a}, \quad \psi^{a}(z)=\sum_{m \in \mathbb{Z}} z^{-m-\frac{1}{2}} \psi_{m}^{a},
$$

this yields an expression for the zero mode of $G(z)$,

$$
-\sqrt{2 \mathrm{k}} G_{0}=\sum_{m \in \mathbb{Z}} \delta_{a b}: \widehat{j}_{m}^{a} \psi_{-m}^{b}:-\frac{1}{3} \sum_{m, n \in \mathbb{Z}} f_{a b c}: \psi_{m}^{a}: \psi_{n}^{b} \psi_{-m-n}^{c}::
$$

which acts in the Ramond sector and is crucial for the NCG interpretation. 


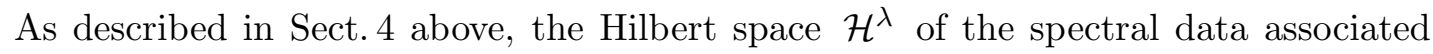
to a maximally symmetric boundary state $\mathscr{D}_{\lambda}$ of weight label $\lambda \in P_{+}^{\mathrm{k}}(\mathfrak{g})$ is chosen to be the subspace of the boundary CFT consisting of the Ramond vacuum sector $\Omega_{\mathrm{R}}$ tensored with the space $\mathcal{H}_{\mathrm{NS}}^{\lambda}$ of Neveau-Schwarz primary boundary fields and their horizontal $\left(\left[\widehat{\mathfrak{g}}_{\widehat{\mathrm{k}}}\right]_{(0)} \cong \mathfrak{g}\right)$ descendants.

We take our algebra of "functions" $\mathcal{A}^{\lambda}$ to be isomorphic to $\mathcal{H}_{\mathrm{NS}}^{\lambda}$ as a vector space (and we will make use of this freely in the following). An element $a \in \mathcal{A}^{\lambda}$ is represented on $\mathcal{H}^{\lambda}$ by the matrix $\mathbf{1}_{D} \otimes a$, acting on $\omega \otimes b \in \mathcal{H}^{\lambda}$ as $a \triangleright(\omega \otimes b):=\omega \otimes a b$, with the matrix product $a b$ defined by the decoupling limit of the boundary OPE [1, 2], which then also defines the product the algebra is endowed with.

We want to restrict (A.7) to the subspace $\mathcal{H}^{\lambda}$ of minimal-energy Ramond states and use it as the generalised Dirac operator of our spectral triple. We first recall that the Ramond vacuum fields $\sigma_{i}, i=1,2, \ldots, D:=2^{\left[\frac{d+1}{2}\right]}$ can be generated from the spin field $\sigma \equiv \sigma_{1}$ of [42] by the action of the free-fermion zero-mode OPE subalgebra $\psi_{0}^{a}$, and that they satisfy

$$
j_{m}^{a} \sigma_{i}=0=\psi_{m}^{a} \sigma_{i}
$$

for $m \neq 0$. Using this, it is not difficult to see that the restriction of the operator (A.7) to $\mathcal{H}^{\lambda}:=\Omega_{R} \otimes \mathcal{H}_{\mathrm{NS}}^{\lambda}$ yields

$$
-\left.\mathrm{i} \sqrt{\frac{\mathrm{k}}{2}} G_{0}\right|_{\mathcal{H}^{\lambda}}=\frac{\mathrm{i}}{2} \delta_{a b} \psi_{0}^{a} \otimes \widehat{j}_{0}^{b}-\frac{\mathrm{i}}{6} f_{a b c} \psi_{0}^{a} \psi_{0}^{b} \psi_{0}^{c} \otimes \mathbf{1}_{D_{\lambda}}
$$

with $D_{\lambda}=\operatorname{dim} \mathcal{H}_{\mathrm{NS}}^{\lambda}$. We now pass to rescaled current modes $-2 \mathrm{i} \widehat{J}_{0}^{a} \equiv \widehat{j}_{0}^{a}$ and rescaled fermion zero modes $\gamma^{a}:=\sqrt{2} \psi_{0}^{a}$, which due to the OPE (A.5) satisfy the relations of the Clifford algebra $\operatorname{Cliff}\left(\mathbb{R}^{d}\right)$,

$$
\left\{\gamma^{a}, \gamma^{b}\right\}=2 \delta^{a b}
$$

Furthermore, we introduce the notation

$$
\widehat{J}_{0}^{a} f=: L_{a}^{\lambda} \triangleright f
$$

for the action of the horizontal-subalgebra generators $\widehat{J}_{0}^{a}$ on $f \in \mathcal{H}_{\mathrm{NS}}^{\lambda}$, as dictated by the relevant boundary OPE

$$
\widehat{J}^{a}\left(x_{1}\right) f\left(x_{2}\right) \sim \frac{1}{x_{1}-x_{2}} L_{a}^{\lambda} \triangleright f\left(x_{2}\right) .
$$

By a slight abuse of notation, we will use the same symbol $\triangleright$ for the action of the $L_{a}^{\lambda}$ on algebra elements. The $L_{a}^{\lambda}$ are then self-adjoint generators of the Lie algebra $\mathfrak{g}$ and accordingly satisfy

$$
\left[L_{a}^{\lambda}, L_{b}^{\lambda}\right]=\mathrm{i} f_{a b c} L_{c}^{\lambda}
$$


The action of $L_{a}^{\lambda}$ is tantamount to an inner derivation ${ }^{14}$

$$
L_{a}^{\lambda} \triangleright f=\left[Y_{a}^{\lambda}, f\right],
$$

where $Y_{a}^{\lambda}$ denote the standard linear combinations of the vector hyperharmonics of $\mathfrak{g}$ compatible with the choice of the basis of $\mathfrak{g}$ in (A.9).

This leads to the following expression for the generalised Dirac operator of the spectral triple (cp [39])

$$
D_{\mathrm{R}}^{\lambda}:=-\left.\mathrm{i} \sqrt{\mathrm{k}} G_{0}\right|_{\mathcal{H}^{\lambda}}=\gamma^{a} \otimes L_{a}^{\lambda}-\frac{\mathrm{i}}{12} f_{a b c} \gamma^{a} \gamma^{b} \gamma^{c} \otimes \mathbf{1}_{D_{\lambda}} .
$$

Note the presence of a torsion term $\frac{\mathrm{i}}{12} f_{a b c} \gamma^{a} \gamma^{b} \gamma^{c} \otimes \mathbf{1}_{D_{\lambda}}$.

We have now established the data of the spectral triple $\mathcal{T}_{\mathrm{WZW}}^{\lambda}=\left(D_{\mathrm{R}}^{\lambda}, \mathcal{A}^{\lambda}, \mathcal{H}^{\lambda}\right)$ that we associate to the boundary WZW model, and will in the following work out those elements of the differential calculus of $\mathcal{T}_{\mathrm{WZW}}^{\lambda}$ which are instrumental for the main body of this paper. The intrinsic definition of a spectral differential calculus is due to Connes [40], many details are e.g. given in 43. We are mainly interested in differential $p$-forms on the non-commutative D-brane world-volumes, in particular for $p=1$ (the gauge field 1-form) and $p=2$ (the gauge field strength 2 -form). On a (representative) of a $p$-form

$$
\omega=\sum_{i \in I} a_{i}^{(0)}\left[D_{\mathrm{R}}^{\lambda}, a_{i}^{(1)}\right]\left[D_{\mathrm{R}}^{\lambda}, a_{i}^{(2)}\right] \cdots\left[D_{\mathrm{R}}^{\lambda}, a_{i}^{(p)}\right]
$$

( $I$ is some index set, $a_{i}^{(n)} \in \mathcal{A}^{\lambda}$ ), the standard definition of the exterior derivative is

$$
\mathrm{d}: \omega \longmapsto \mathrm{d} \omega \equiv D_{\mathrm{R}}^{\lambda} \omega-(-1)^{p} \omega D_{\mathrm{R}}^{\lambda}
$$

and reduces to the explicit formula

$$
\mathrm{d} \omega=\sum_{i \in I}\left[D_{\mathrm{R}}^{\lambda}, a_{i}^{(0)}\right]\left[D_{\mathrm{R}}^{\lambda}, a_{i}^{(1)}\right]\left[D_{\mathrm{R}}^{\lambda}, a_{i}^{(2)}\right] \cdots\left[D_{\mathrm{R}}^{\lambda}, a_{i}^{(p)}\right]
$$

this uses the Lichnerowicz formula which relates $\left(D_{\mathrm{R}}^{\lambda}\right)^{2}$ to the Virasoro Hamiltonian $L_{0}$, as explicited (in the bulk case) in [39]. The definitions of differential 0-forms

$$
\widetilde{\Omega}_{D_{\mathrm{R}}^{\lambda}}^{0}\left(\mathcal{A}^{\lambda}\right)=\mathcal{A}^{\lambda}
$$

and of differential 1-forms

$$
\widetilde{\Omega}_{D_{\mathrm{R}}^{\lambda}}^{1}\left(\mathcal{A}^{\lambda}\right)=\left\{\gamma^{a} \otimes a_{a} \mid a_{a}=\sum_{i \in I} a_{i}^{(0)}\left[Y_{a}^{\lambda}, a_{i}^{(1)}\right], \quad a_{i}^{(0),(1)} \in \mathcal{A}^{\lambda}\right\}
$$

\footnotetext{
${ }^{14}$ The statement can readily be verified in the hyperharmonic basis of the algebra of functions on the fuzzy conjugacy class $\mathscr{D}_{\lambda}$. First, it is elementary to check that A.10 yields an action of $\mathfrak{g}$ on the vector multiplet itself (the coordinate "functions"), concordant with the representation label carried by the latter. Then, all we need to extend this action to homogeneous polynomials in the coordinates, reproducing higher hyperharmonics, is the primitive coalgebra structure on $\mathfrak{g}$.
} 
are straightforward, but that of differential 2-forms is somewhat more involved [40]: From the set of all

$$
\omega=\sum_{i \in I} a_{i}^{(0)}\left[\mathcal{D}_{\mathrm{R}}^{\lambda}, a_{i}^{(1)}\right]\left[\mathcal{D}_{\mathrm{R}}^{\lambda}, a_{i}^{(2)}\right]
$$

we have to project out the so-called "junk forms", i.e. operators of the kind

$$
\eta:=\sum_{i \in I}\left[\mathcal{D}_{\mathrm{R}}^{\lambda}, a_{i}^{(0)}\right]\left[\mathcal{D}_{\mathrm{R}}^{\lambda}, a_{i}^{(1)}\right] \quad \text { such that } \sum_{i \in I} a_{i}^{(0)}\left[\mathcal{D}_{\mathrm{R}}^{\lambda}, a_{i}^{(1)}\right] \equiv 0 .
$$

Using the explicit form of the Ramond-Dirac operator (A.11) and the Clifford algebra (A.8), we see that the junk 2-forms are

$$
\eta=-\sum_{i \in I} \delta^{a b} \cdot \mathbf{1}_{D} \otimes a_{i}^{(0)}\left(L_{a}^{\lambda} L_{b}^{\lambda} \triangleright a_{i}^{(1)}\right) .
$$

The operator acting on $a_{i}^{(1)}$ in (A.13) is just the Laplacian on the fuzzy conjugacy class associated with $\mathcal{D}_{\lambda}$. This observation, together with the fact that the "junk forms" furnish a left $\mathcal{A}^{\lambda}$-module, allows to show that

$$
\widetilde{\Omega}_{D_{\mathrm{R}}^{\lambda}}^{2}\left(\mathcal{A}^{\lambda}\right)=\left\{\gamma^{a b} \otimes f_{a b} \mid f_{a b}=\mathrm{i} \sum_{i \in I} a_{i}^{(0)}\left[Y_{[a}^{\lambda}, a_{i}^{(1)}\right]\left[Y_{b]}^{\lambda}, a_{i}^{(2)}\right], \quad a_{1}^{(0),(1),(2)} \in \mathcal{A}^{\lambda}\right\}
$$

where $\gamma^{a b}:=-\frac{i}{2}\left[\gamma^{a}, \gamma^{b}\right]$.

As a sample computation to indicate the relevance of projecting out "junk forms", let us look at the $\mathrm{d} A$-piece of the Yang-Mills field strength $F$ : Starting from the 1-form

$$
A=\gamma^{a} \otimes \sum_{i \in I} a_{i}^{(0)}\left(L_{a}^{\lambda} \triangleright a_{i}^{(1)}\right)
$$

we compute the 2 -form part of $\mathrm{d} A$ by projecting out components of the type (A.12), which results in the unfamiliar term $f_{a b c} A_{c}$ in $F_{a b}$ from (1.3), using that the $L_{a}^{\lambda}$ furnish a representation of the Lie algebra $\mathfrak{g}$,

$$
\begin{aligned}
{[\mathrm{d} A]_{2 \text {-form }} } & \equiv\left(\sum_{i \in I}\left[\mathcal{D}_{\mathrm{R}}^{\lambda}, a_{i}^{(0)}\right]\left[\mathcal{D}_{\mathrm{R}}^{\lambda}, a_{i}^{(1)}\right]\right)_{2 \text {-form }}=\sum_{i \in I} \gamma^{a b} \otimes \mathrm{i}\left(L_{a}^{\lambda} \triangleright a_{i}^{(0)}\right)\left(L_{b}^{\lambda} \triangleright a_{i}^{(1)}\right)= \\
& =\gamma^{a b} \otimes \frac{1}{2}\left(\mathrm{i} L_{a}^{\lambda} \triangleright A_{b}-\mathrm{i} L_{b}^{\lambda} \triangleright A_{a}+f_{a b c} A_{c}\right) .
\end{aligned}
$$

The expressions for 1-forms and 2-forms given above are still slightly unwieldy. Arguments given in [45, 46] for rather general non-commutative spectral data over full matrix algebras show that the spaces of $p$-forms are in fact free modules over the algebra of functions $\mathcal{A}^{\lambda}$,

$$
\widetilde{\Omega}_{D_{\mathrm{R}}^{\lambda}}^{p}\left(\mathcal{A}^{\lambda}\right) \cong\left\{\gamma^{a_{1} a_{2} \ldots a_{p}} \otimes f_{a_{1} a_{2} \ldots a_{p}} \mid \quad f_{a_{1} a_{2} \ldots a_{p}} \in \mathcal{A}^{\lambda}\right\},
$$

where the $\gamma^{a_{1} a_{2} \ldots a_{p}}$ are totally antisymmetric products of $\gamma^{a}$. 


\section{References}

[1] A.Yu. Alekseev, A. Recknagel, and V. Schomerus, "Non-commutative world-volume geometries: branes on $S U(2)$ and fuzzy spheres", JHEP 9909 (1999) 023 hep-th/9908040.

[2] A.Yu. Alekseev, A. Recknagel, and V. Schomerus, "Brane dynamics in background fluxes and non-commutative geometry", JHEP 0005 (2000) 010 hep-th/0003187.

[3] N. Ishibashi, H. Kawai, Y. Kitazawa, and A. Tsuchiya, "A large- $N$ reduced model as superstring", Nucl. Phys. B498 (1997) 467-491 hep-th/9612115.

[4] T. Yoneya, "Schild action and space-time uncertainty principle in string theory", Prog. Theor. Phys. 97 (1997) 949-962 hep-th/9703078]; "D-particles, D-instantons, and a space-time uncertainty principle in string theory", in Seoul 1997, Recent developments in nonperturbative quantum field theory, Y.M. Chop and M. Virasoro (eds.), World Scientific, 1998 hep-th/9707002.

[5] A.Yu. Alekseev and V. Schomerus, "D-branes in the WZW model", Phys. Rev. D60 (1999) 061901 hep-th/9812193].

[6] K. GawȨDZKI, "Conformal field theory: a case study" hep-th/9904145.

[7] C. KLIMČík, "A nonperturbative regularization of the supersymmetric Schwinger model", Commun. Math. Phys. 206 (1999) 567-586 hep-th/9903112.

[8] R. C. Myers, "Dielectric-branes", JHEP 9912 (1999) 022 hep-th/9910053.

[9] C. Bachas, M.R. Douglas, and C. Schweigert, "Flux stabilization of D-branes", JHEP 0005 (2000) 048 hep-th/0003037.

[10] J. PAWEecZYK, " $S U(2)$ WZW D-branes and their non-commutative geometry from DBI action", JHEP 0008 (2000) 006 hep-th/0003057.

[11] P. Bordalo, S. Ribault, and C. Schweigert, "Flux stabilization in compact groups", JHEP 0110 (2001) 036 hep-th/0108201.

[12] S. Fredenhagen, "Dynamics of D-branes in curved backgrounds", Ph.D. thesis, Humboldt Universität, 2002 http://edoc.hu-berlin.de/dissertationen/ fredenhagen-stefan-2002-09-16/PDF/Fredenhagen.pdf].

[13] S. Fredenhagen and V. Schomerus, "Branes on group manifolds, gluon condensates, and twisted K-theory", JHEP 0104 (2001) 007 hep-th/0012164.

[14] A.Yu. Alekseev, S. Fredenhagen, T. Quella, and V. Schomerus, "Non-commutative gauge theory of twisted D-branes", Nucl. Phys. B646 (2002) 127 hep-th/0205123.

[15] S. Monnier, "D-branes in Lie groups of rank > 1", JHEP 0508 (2005) 062 hep-th/0507159.

[16] I. Affleck And A.W.W. Ludwig, "The Kondo effect, conformal field theory and fusion rules", Nucl. Phys. B352 (1991) 849-862; "Critical theory of overscreened Kondo fixed points", Nucl. Phys. B60 (1991) 641-696; "Universal noninteger 'ground state degeneracy' in critical quantum systems", Phys. Rev. Lett. 67 (1991) 161-164.

[17] A. Schild, "Classical null strings", Phys. Rev. D16 (1977) 1722-1726.

[18] R.R. Suszek, "From higher to twisted gauge fields via deformed $\sigma$-models", in preparation.

[19] J.R. Hoppe, "Quantum theory of a massless relativistic surface and a two-dimensional bound state problem", Ph.D. thesis, Massachusetts Institute of Technology, 1982. 
[20] O.A. Soloviev, "On the Schild action for $D=0$ and $D=1$ strings", Mod. Phys. Lett. A13 (1998) 2415-2426 hep-th/9708021.

[21] E. Braaten, T.L. Curtright, and C.K. Zachos, "Torsion and geometrostasis in nonlinear sigma models", Nucl. Phys. B260 (1985) 630-688.

[22] L. Cornalba and R. Schiappa, "Nonassociative star product deformations for D-brane world-volumes in curved backgrounds", Commun. Math. Phys. 225 (2002) 33-66 hep-th/0101219.

[23] P. Bordalo, L. Cornalba, and R. Schiappa, "Towards quantum dielectric branes: Curvature corrections in abelian beta function and nonabelian Born-Infeld action", Nucl. Phys. B710 (2005) 189-254 hep-th/0409017].

[24] T.L. Curtright and C.K. Zachos, "Geometry, topology and supersymmetry in nonlinear models", Phys. Rev. Lett. 53 (1984) 1799-1801.

[25] V.C. de Andrade, L.C.T. Guillen, and J.G. Pereira "Teleparallel gravity: an overview", in The Ninth Marcel Grossmann Meeting: On Recent Developments in Theoretical and Experimental General Relativity, Gravitation and Relativistic Field Theories, Proceedings of the MGIX MM Meeting, The University of Rome "La Sapienza" 2-8 July 2000, V.G. Gurzadyan, R.T. Jantzen, and R. Ruffini (eds.), World Scientific, 2002 gr-qc/0011087.

[26] R. Aldrovandi And J.G. Pereira, "An introduction to teleparallel gravity", Lecture notes Universidad de Concepción, 2005.

[27] F. Gronwald and F.W. Hehl, "On the gauge aspects of gravity", in Quantum Gravity: International School of Cosmology and Gravitation XIV Course : 80th Birthday Dedication to Peter G. Bergmann : Erice, Italy 11-19 May, 1995, P.G. Bergmann, V. de Sabbata, and H.-J. Treder (eds.), World Scientific, 1996 [gr-qc/9602013].

[28] K. GAwȨDZKI, "Topological actions in two-dimensional quantum field theories", in Non-Perturbative Quantum Field Theory, G. 't Hooft, A. Jaffe, G. Mack, P.K. Mitter, and R. Stora (eds.), Plenum Press, 1988.

[29] K. Gawȩdzki and N. Reis, "WZW branes and gerbes", Rev. Math. Phys. 14 (2002) 1281-1334 hep-th/0205233.

[30] K. GAWȨDZKI, "Abelian and non-Abelian branes in WZW models and gerbes", Commun. Math. Phys. 258 (2005) 23-73 hep-th/0406072].

[31] O. Alvarez, "Topological quantization and cohomology", Commun. Math. Phys. 100 (1985) 279-309.

[32] J.-L. Brylinski, Loop spaces, characteristic classes and geometric quantization, Progress in Mathematics, vol. 107, Birkhäuser, 1993.

[33] J. Giraud, Cohomologie non abélienne, Grundlehren der Mathematischen Wissenschaften, vol. 179, Springer, 1971.

[34] M.K. Murray, "Bundle gerbes", J. London Math. Soc. 54 (1996) 403-416 dg-ga/9407015.

[35] M.K. Murray and D. Stevenson, "Bundle gerbes: stable isomorphism and local theory", J. London Math. Soc. 62 (2000) 925-937 math.DG/9908135.

[36] S. Johnson, "Constructions with bundle gerbes", Ph.D. thesis, University of Adelaide, 2002 math.DG/0312175. 
[37] E. Witten, "Non-abelian bosonization in two dimensions", Commun. Math. Phys. 92 (1984) 455-472.

[38] A. Kapustin, "D-branes in a topologically nontrivial B-field", Adv. Theor. Math. Phys. 4 (2000) 127-154 hep-th/9909089.

[39] J. Fröhlich And K. GawȨDZKI, "Conformal field theory and geometry of strings", in Vancouver 1993, Proceedings, Mathematical quantum theory I: Field Theory and Many-Body Theory, J. Feldman, R. Froese, and L.M. Rosen (eds.), CRM Proceedings \& Lecture Notes, vol. 7, American Mathematical Society, 1994 hep-th/9310187.

[40] A. Connes, Noncommutative Geometry, Academic Press, 1994.

[41] D. Roggenkamp and K. Wendland, "Limits and degenerations of unitary conformal field theories", Commun. Math. Phys. 251 (2004) 589-643 hep-th/0308143.

[42] J. Fuchs, "Superconformal Ward identities and the WZW model", Nucl. Phys. B286 (1987) 455-484; "More on the super WZW theory", Nucl. Phys. B318 (1989) 631-654.

[43] J. Fröhlich, O. Grandjean, and A. Recknagel, "Supersymmetric quantum theory and (non-commutative) differential geometry", Commun. Math. Phys. 193 (1998) 527-594 hep-th/9612205; "Supersymmetric quantum theory, non-commutative geometry, and gravitation", in NATO Advanced Study Institute: Les Houches Summer School On Theoretical Physics, Session 64: Quantum Symmetries, A. Connes, K. Gawędzki, and J. Zinn-Justin (eds.), North-Holland, 1998 hep-th/9706132]; "Supersymmetric quantum theory and non-commutative geometry", Commun. Math. Phys. 203 (1999) 119-184 hep-th/9807006.

[44] O. Grandjean, "Non-commutative differential geometry", Ph.D. thesis, Eidgenössische Technische Hochschule, Zürich, 1997.

[45] J. Madore, An Introduction to Noncommutative Differential Geometry and its Physical Applications, London Mathematical Society Lecture Note Series, vol. 257, Cambridge University Press, 1995.

[46] J. Gratus, "Non-commutative differential geometry, and the matrix representations of generalised algebras", J. Geom. Phys. 25 (1998) 227-244 q-alg/9703034.

[47] V.C. de Andrade and J.G. Pereira, "Torsion and the electromagnetic field", Int. J. Mod. Phys. D8 (1999) 141-151 gr-qc/9708051.

[48] E. S. Fradkin and A. A. Tseytlin, "Effective field theory from quantized strings", Phys. Lett. B158 (1985) 316-322; "Effective action approach to superstring theory", Phys. Lett. B160 (1985) 69-76; "Nonlinear electrodynamics from quantized strings", Phys. Lett. B163 (1985) 123-130; "Quantum string theory effective action", Nucl. Phys. B261 (1985) 1-27.

[49] D.H. Friedan, "Nonlinear Models in $2+\epsilon$ Dimensions", Ph.D. thesis, Lawrence Berkeley Laboratory, Berkeley, 1980, Annals Phys. 163 (1985) 318-419.

[50] K. GAWȨDZKI, "Lectures on conformal field theory", 1996-97 Quantum Field Theory program at IAS, Princeton, available at http://www.math.ias.edu/QFT/fall/index.html.

[51] G.A. Vilkovisky, "The unique effective action in quantum field theory", Nucl. Phys. B234 (1984) 125-137.

[52] A.O. Barvinsky and G.A. Vilkovisky, "The generalized Schwinger-DeWitt technique in gauge theories and quantum gravity", Phys. Rep. 119 (1985) 1-74. 
[53] B.S. DeWitT, "The Effective Action", in Quantum Field Theory and Quantum Statistics: Quantum Statistics and Methods of Field Theory, I.A. Batalin, C.J. Isham, and G.A. Vilkovisky (eds.), Hilger, 1987.

[54] J. Pawęczyk and H. Steinacker, "Matrix description of D-branes on 3-spheres", JHEP 0112 (2001) 018 hep-th/0107265; "A quantum algebraic description of D-branes on group manifolds", Nucl. Phys. B638 (2002) 433-458 [hep-th/0203110].

[55] J. Paweeczyk and R.R. Suszek, "Brane bulk couplings and condensation from REA fusion", JHEP 0604 (2006) 009 hep-th/0503240.

[56] P. Castro-Villarreal, R. Delgadillo-Blando, and B. Ydri, "A gauge invariant UV-IR mixing and the corresponding phase transition for $U(1)$ fields on the fuzzy sphere", Nucl. Phys. B704 (2005) 111-153 hep-th/0405201.

[57] C.-S. Chu, J. Madore, and H. Steinacker, "Scaling limits of the fuzzy sphere at one loop", JHEP 0108 (2001) 038 hep-th/0106205.

[58] B. Kostant, "A cubic Dirac operator and the emergence of Euler number multiplets of representations for equal rank subgroups", Duke Math. J. 100 (1999) 447-501.

[59] L. BRink AND P. RAmond, "Dirac equations, light cone supersymmetry, and superconformal algebras" hep-th/9908208.

[60] A. Connes, M.R. Douglas, and A. Schwarz, "Noncommutative geometry and Matrix theory: compactification on tori", JHEP 9802 (1998) 003 [hep-th/9711162].

[61] N. Seiberg and E. Witten, "String theory and non-commutative geometry", JHEP 9909 (1999) 032 [hep-th/9908142].

[62] A. Schwarz, "Morita equivalence and duality", Nucl. Phys. B534 (1998) 720-738 hep-th/9805034.

[63] D. Brace, B. Morariu, and B. Zumino, "Dualities of the Matrix Model from T-duality of the type II string", Nucl. Phys. B545 (1999) 192-216 hep-th/9810099;; "T-duality and Ramond-Ramond backgrounds in the Matrix Model", Nucl. Phys. B549 (1999) 181-193 hep-th/9811213.

[64] A. Konechny And A. Schwarz, "BPS states on noncommutative tori and duality", Nucl. Phys. B550 (1999) 561-584 hep-th/9811159.

[65] B. Pioline And A. Schwarz, "Morita equivalence and T-duality (or $B$ versus $\Theta$ )", JHEP 9908 (1999) 021 hep-th/9908019. 